\author{
Andrzej Pietruszczakio
}

TOMASZ JARMUŻEK

\section{Pure Modal Logic of Names and Tableau Systems}

\begin{abstract}
By a pure modal logic of names (PMLN) we mean a quantifier-free formulation of such a logic which includes not only traditional categorical, but also modal categorical sentences with modalities de re and which is an extension of Propositional Logic. For categorical sentences we use two interpretations: a "natural" one; and Johnson and Thomason's interpretation, which is suitable for some reconstructions of Aristotelian modal syllogistic (Johnson in Notre Dame J Form Logic 30(2):271-284, 1989; Thomason in J Philos Logic 22(2):111-128, 1993 and J Philos Logic 26:129-141, 1997. In both cases we use Johnson-like models (1989). We also analyze different kinds of versions of PMLN, for both general and singular names. We present complete tableau systems for the different versions of PMLN. These systems enable us to present some decidability methods. It yields "strong decidability" in the following sense: for every inference starting with a finite set of premises (resp. every syllogism, every formula) we can specify a finite number of steps to check whether it is logically valid. This method gives the upper bound of the cardinality of models needed for the examination of the validity of a given inference (resp. syllogism, formula).

Keywords: Pure modal logic of names, Semantics, Tableaus, Decidability, Modal syllogistic.
\end{abstract}

\title{
Introduction
}

By a pure modal logic of names (PMLN) we mean a quantifier-free formulation of such a logic, in which to traditional categorical sentences we add also modal categorial sentences with modalities de re and which is an extension of Propositional Logic. In this paper we present complete tableau systems for this logic as well as for modal syllogisms in Johnson-like models for categorial sentences. Johnson [4] and Thomason [14,15] used a natural interpretation for general sentences of the form 'Every $a$ is necessarily $b$ ' and 'No $a$ is possibly $b$ '. In Section 8 we describe pure modal logics for both general and singular names and some tableau systems for the logics.

Presented by Andrzej Indrzejczak; Received June 26, 2017 
We will in this paper take a natural interpretation of these four kinds of sentence, since we do not aim to reconstruct Aristotelian syllogistic. However, to reconstruct Aristotelian modal syllogistic, in $[4,14,15]$ the authors have assumed unnatural interpretations of particular sentences of the form: 'Some $a$ is necessarily $b$ ' and 'Some $a$ is not possibly $b$ ', and as a consequence also of general sentences of the form: 'Every $a$ is possibly $b$ ' and 'No $a$ is necessarily $b$ ' (see Section 9). In the last part of the paper the unnatural interpretation is discussed and may be compared with the former results. Summing up, the general aim of the paper is to examine natural as well as unnatural interpretations of modal syllogism in the light of modern tableau tools.

In this paper we present a special kind of tableau, which we call a "minimax" tableau. Mini-max tableaus involve a very low number of proof steps. They give us a decidability method for PMLN (as well as for modal syllogistic) which yields what we call "strong decidability". It means that for every inference starting with a finite set of premises (resp. every syllogism, every formula) we can specify a finite number of steps to check whether it is logically valid. This method gives also the upper bound of the cardinality of models needed for the examination of the validity of a given inference (resp. syllogism, formula).

For syllogisms, as in [3], we can also provide another decidability method which estimates a limit of the length of a tableau for a given syllogism. This paper is both a significant extension of [3] and a modification of it.

\section{Pure Modal Logic of Names and Modal Syllogisms}

\subsection{Syntax}

Formulas of modal syllogisms. We assume a countably infinite set GL which contains schematic general name letters: $a_{0}, a_{1}, a_{2}, a_{3}, \ldots$ (for the first three of these letters we will use abbreviations: ' $a$ ', ' $b$ ', and ' $c$ ', respectively). We assume that the letters from GL represent various general names.

Following Thomason $[14,15]$ a modal syllogistic formula is an expression of any of the following forms: $\mathrm{A} \alpha \beta, \mathrm{I} \alpha \beta, \mathrm{E} \alpha \beta, \mathrm{O} \alpha \beta, \mathrm{A}^{\square} \alpha \beta, \mathrm{A}^{\diamond} \alpha \beta, \mathrm{I}^{\square} \alpha \beta$, $\mathrm{I}^{\diamond} \alpha \beta, \mathrm{E}^{\square} \alpha \beta, \mathrm{E}^{\diamond} \alpha \beta, \mathrm{O}^{\square} \alpha \beta, \mathrm{O}^{\diamond} \alpha \beta$, for $\alpha, \beta \in \mathbf{G L}$. The notation reflects a way to read non-modal and apodeictic propositions, respectively: A $a b$ : $b$ belongs to every $a$, E $a b: b$ belongs to no $a$, I $a b: b$ belongs to some $a, \mathrm{O} a b: b$ does not belong to some $a ; A^{\square} a b: b$ of necessity belongs to every $a ; \mathrm{E}^{\square} a b: b$ of necessity belongs to no $a$; $\mathrm{I}^{\square} a b: b$ belongs of necessity to some $a ; \mathrm{O}^{\square} a b: b$ of necessity does not belong to some $a$ (see, e.g., [1] and [7, p. 10]). Similar 
readings hold for formulas with ' $\diamond$ ', where 'of necessity' is replaced with 'of possibility'.

Let For $_{\text {syll }}$ be the set of all syllogistic formulas. All formulas with symbols A, I, E, or $\mathrm{O}$ we will call non-modal. For any $\bullet \in\{$ blank, $\square, \diamond\}$ all formulas with $\mathrm{I}^{\bullet}$ or $\mathrm{O}^{\bullet}$ we will call particular (for short: $p$-formulas), and all formulas with $A^{\bullet}$ or $E^{\bullet}$ we will call general (for short: $g$-formulas).

We present English glosses for modal syllogistic formulas under a formal interpretation which reflects a natural way of understanding them [see, e.g., 7, pp. 10-35]. Moreover, the interpretations are expressed by translations into formulas of monadic first-order modal logic: ${ }^{1}$

1. A $a b$ : Every $a$ is $b$

$A^{\square} a b$ : Every $a$ must be $b$, Every $a$ is necessarily $b$

$$
\begin{array}{r}
\forall_{x}(a x \supset b x) \\
\forall_{x}(a x \supset \square b x) \\
\forall_{x}(a x \supset \diamond b x) \\
\neg \exists_{x}(a x \wedge b x) \\
\neg \exists_{x}(a x \wedge \diamond b x) \\
\neg \exists_{x}(a x \wedge \square b x) \\
\exists_{x}(a x \wedge b x) \\
\exists_{x}(a x \wedge \square b x) \\
\exists_{x}(a x \wedge \diamond b x) \\
\exists_{x}(a x \wedge \neg b x) \\
\exists_{x}(a x \wedge \square \neg b x) \\
\exists_{x}(a x \wedge \neg \diamond b x) \\
\exists_{x}(a x \wedge \diamond \neg b x) \\
\exists_{x}(a x \wedge \neg \square b x)
\end{array}
$$$$
A^{\diamond} a b \text { : Every } a \text { may be } b \text {, Every } a \text { is possibly } b
$$

2. E $a b$ : No $a$ is $b$

$\mathrm{E}^{\square} a b$ : No $a$ may be $b$, No $a$ is possibly $b$

$\mathrm{E}^{\diamond} a b$ : No $a$ must be $b$, No $a$ is necessarily $b$

3. I $a b$ : Some $a$ is $b$

$\mathrm{I}^{\square} a b$ : Some $a$ must be $b$, Some $a$ is necessarily $b$

$\mathrm{I}^{\diamond} a b$ : Some $a$ may be $b$, Some $a$ is possibly $b$

4. Oab: Some $a$ is not $b$

$\mathrm{O}^{\square} a b$ : Some $a$ must not be $b$

Some $a$ is not possibly $b$

$\mathrm{O}^{\diamond} a b$ : Some $a$ may not be $b$

Some $a$ is not necessarily $b$

Syllogisms. By syllogism we mean any pair $\langle\Psi, \varphi\rangle$, where $\Psi$ is a non-empty finite subset ${ }^{2}$ of For $_{\text {syll }}$ (for short: $\Psi \in \mathcal{P}_{\text {fin }}\left(\mathbf{F o r}_{\text {syll }}\right) \backslash\{\emptyset\}$ ) and $\varphi \in$ For $_{\text {syll }}{ }^{3}$

Formulas of PMLN. All formulas of PMLN are built with members of

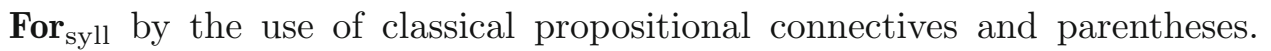
These formulas are members of For which is the smallest set such that:

\footnotetext{
${ }^{1}$ Formulas of monadic first-order modal logic are defined in a standard way: ' $a x$ ' and ' $b x$ ' stands for ' $x$ is $a$ ' and ' $x$ is $b$ ', respectively; ' $\square$ ' and ' $\nabla$ ' stand for the de dicto operators 'it is necessary that' and 'it is possible that', respectively.

${ }^{2}$ For any set $S$ let $\mathcal{P}(S)$ (resp. $\mathcal{P}_{\text {fin }}(S)$ ) be the family of all (resp. finite) subsets of $S$.

${ }^{3}$ The denotation of the term 'syllogism' is in our paper wider than in [1].
} 
- For $_{\text {syll }} \subseteq$ For;

- if $\varphi \in$ For, than $\ulcorner\neg \varphi\urcorner \in$ For;

- if $\varphi, \psi \in$ For, than $\ulcorner(\varphi \circ \psi)\urcorner \in$ For, where $\circ \in\{\wedge, \vee, \supset, \equiv\}$.

For any $\Phi \in \mathcal{P}($ For $)$ let $\mathbf{G L}(\Phi)$ be the set of letters from GL which occur in at least one formula of $\Phi$.

\subsection{Semantics}

Models and the notion of truth for For. A model ${ }^{4}$ for For is any quadruple $\left\langle D, d, d^{\square}, d^{\diamond}\right\rangle$, where $D$ is a set (domain; possible empty) and $d, d^{\square}, d^{\diamond}$ are functions from $\mathbf{G L}$ into $\mathcal{P}(D)$ satisfying the following condition:

$(\star) \quad d^{\square}(\alpha) \subseteq d(\alpha) \subseteq d^{\diamond}(\alpha)$, for any $\alpha \in \mathbf{G L}$.

Intuitively, $d(a)$ is the denotation of $a$ in $\mathfrak{M}$, i.e., it is the set of all objects which are $a$. Moreover, $d^{\square}(a)$ (resp. $d^{\diamond}(a)$ ) is the set of all objects which are necessarily (resp. possibly) $a .^{5}$

Let Mod be the class of all models for For. The empty model $\mathfrak{M}_{\emptyset}$ is the only model with the empty universe. This model has also empty denotation functions, i.e., $d(\alpha)=d^{\square}(\alpha)=d^{\diamond}(\alpha)=\emptyset$, for any $\alpha \in \mathbf{G L}$.

We use the following interpretation of formulas from For in model $\mathfrak{M}=$ $\left\langle D, d, d^{\square}, d^{\diamond}\right\rangle$, for all $\alpha, \beta \in \mathbf{G L}, \varphi, \psi \in$ For, and $\bullet \in\{$ blank, $\square, \diamond\}$ :

$\mathfrak{M} \vDash A^{\bullet} \alpha \beta$ iff $d(\alpha) \subseteq d^{\bullet}(\beta)$,

$\mathfrak{M} \vDash \mathrm{E}^{\bullet} \alpha \beta$ iff $d(\alpha) \cap d^{*}(\beta)=\emptyset$,

where $*=$ blank, $\diamond, \square$, for $\bullet=$ blank, $\square, \diamond$, respectively,

$\mathfrak{M} \vDash \mathrm{I}^{\bullet} \alpha \beta$ iff $d(\alpha) \cap d^{\bullet}(\beta) \neq \emptyset$,

$\mathfrak{M} \vDash \mathrm{O}^{\bullet} \alpha \beta$ iff $d(\alpha) \nsubseteq d^{*}(\beta)$,

where $*=$ blank, $\diamond, \square$, for $\bullet=$ blank, $\square, \diamond$, respectively,

$\mathfrak{M} \vDash \neg \varphi$ iff $\mathfrak{M} \not \models \varphi$,

$\mathfrak{M} \vDash(\varphi \wedge \psi)$ iff $\mathfrak{M} \vDash \varphi$ and $\mathfrak{M} \vDash \psi$,

$\mathfrak{M} \vDash(\varphi \vee \psi)$ iff $\mathfrak{M} \vDash \varphi$ or $\mathfrak{M} \vDash \psi$,

$\mathfrak{M} \vDash(\varphi \supset \psi)$ iff $\mathfrak{M} \not \models \varphi$ or $\mathfrak{M} \vDash \psi$,

$\mathfrak{M} \vDash(\varphi \equiv \psi)$ iff either $\mathfrak{M} \vDash \varphi$ and $\mathfrak{M} \vDash \psi$, or $\mathfrak{M} \not \models \varphi$ and $\mathfrak{M} \not \models \psi$.

\footnotetext{
${ }^{4}$ The notion of model is based on [15], where Thomason adopted Johnson's notion from [4] and his own notion from [14].

${ }^{5}$ All pairs of the form $\langle D, d\rangle$ are models for non-modal formulas [see, e.g., 8-11].
} 
If $\mathfrak{M} \vDash \varphi$ then we say that the formula $\varphi$ is true in $\mathfrak{M}$. Otherwise, i.e. $\mathfrak{M} \not \models \varphi$, we say that it is false in $\mathfrak{M}$. If for any $\psi$ from $\Psi \subseteq$ For we have $\mathfrak{M} \vDash \psi$, then we write $\mathfrak{M} \vDash \Psi$.

Note that the empty model $\mathfrak{M}_{\emptyset}$ plays the same role as for any set $D$ the model $\mathfrak{M}_{D, \emptyset}:=\langle D, \alpha \mapsto \emptyset, \alpha \mapsto \emptyset, \alpha \mapsto \emptyset\rangle$ which has empty denotation functions.

Lemma 1.1. For any set $D$ and any $\varphi \in$ For: $\mathfrak{M}_{D, \emptyset} \vDash \varphi$ iff $\mathfrak{M}_{\emptyset} \vDash \varphi$.

Logical entailment and equivalence. Logically valid formulas and syllogisms. For all $\Psi \in \mathcal{P}($ For $)$ and $\varphi \in$ For, we say that $\Psi$ logically entails $\varphi$ (we write: $\Psi \models \varphi$ ) iff there is no model $\mathfrak{M}$ in Mod such that $\mathfrak{M} \vDash \Psi$ and $\mathfrak{M} \not \models \varphi$. If $\Psi=\left\{\psi_{1}, \ldots, \psi_{n}\right\} \quad(n>0)$, then we write: $\psi_{1}, \ldots, \psi_{n}=\varphi$. Formulas $\varphi, \psi \in$ For are logically equivalent (we write: $\varphi \models \mid \psi$ ) iff $\varphi \models \psi$ and $\psi \models \varphi$. Moreover, a formula $\varphi$ is logically valid (we write: $=\varphi$ ) iff $\emptyset \models \varphi$.

Note that, by Lemma 1.1, we obtain:

Claim 1.2. For any $\Psi \in \mathcal{P}$ (For) and $\varphi \in$ For: $\Psi=\varphi$ iff there is no nonempty model $\mathfrak{M}$ in Mod such that $\mathfrak{M} \vDash \Psi$ and $\mathfrak{M} \not \models \varphi$.

We say that a syllogism $\langle\Psi, \varphi\rangle$ is logically valid iff $\Psi \models \varphi$. Of course, a syllogism $\left\langle\left\{\psi_{1}, \ldots, \psi_{n}\right\}, \varphi\right\rangle$ is logically valid iff the formula $\left\ulcorner\psi_{1} \supset(\ldots \supset\right.$ $\left.\left.\left(\psi_{n} \supset \varphi\right)\right)\right\urcorner$ (resp. $\left.\left\ulcorner\left(\psi_{1} \wedge \cdots \wedge \psi_{n}\right) \supset \varphi\right\urcorner\right)$ is logically valid.

Note that for categorical sentences we obtain:

Claim 1.3. For all $\alpha, \beta \in \mathbf{G L}$ :

1. $\mathrm{A}^{\bullet} \alpha \beta \models \neg \mathrm{O}^{*} \alpha \beta$ and $\mathrm{E}^{\bullet} \alpha \beta \models \neg \mathrm{I}^{*} \alpha \beta$, where $*=$ blank, $\diamond, \square$, for $\bullet=$ blank, $\square, \diamond$, respectively.

2. For any $\mathrm{X} \in\{\mathrm{A}, \mathrm{I}, \mathrm{E}, \mathrm{O}\}: \mathrm{X}^{\square} \alpha \beta \models \mathrm{X} \alpha \beta \models \mathrm{X}^{\diamond} \alpha \beta$.

3. $\mathrm{I} \alpha \beta \models \mid \mathrm{I} \beta \alpha$ and $\mathrm{E} \alpha \beta \models \mathrm{E} \beta \alpha$.

4. $\mathrm{I} \alpha \alpha \models \mid \mathrm{I}^{\diamond} \alpha \alpha$.

5. $\mathrm{I}^{\bullet} \alpha \beta=\mathrm{I} \alpha \alpha$ and $\mathrm{O}^{\bullet} \alpha \beta=\mathrm{I} \alpha \alpha$, for any $\bullet \in\{$ blank, $\square, \diamond\}$.

6. $\mid=\mathrm{A} \alpha \alpha$ and $\mid=\mathrm{A}^{\diamond} \alpha \alpha$. So also $\models \neg \mathrm{O} \alpha \alpha$ and $\models \neg \mathrm{O}^{\square} \alpha \alpha$.

7. $\forall A^{\square} \alpha \alpha$ and $\forall \mathrm{I}^{\bullet} \alpha \alpha$, for any $\bullet \in\{$ blank, $\square, \diamond\}$.

8. $\mathrm{A}^{\bullet} \alpha \beta \not=\mathrm{I}^{\bullet} \alpha \beta, \mathrm{E}^{\bullet} \alpha \beta \not \mathrm{O}^{\bullet} \alpha \beta$, for any $\bullet \in\{$ blank, $\square, \diamond\}$.

9. $\mathrm{I}^{\bullet} \alpha \beta \not=\mathrm{I}^{\bullet} \beta \alpha$ and $\mathrm{E}^{\bullet} \alpha \beta \not \models \mathrm{E} \bullet \beta \alpha$, for any $\bullet \in\{\square, \diamond\}$.

Logical contradiction. We say that $\varphi, \psi \in$ For are logically contradictory iff $\varphi \models \neg \psi$. Of course, $\varphi$ and $\neg \varphi$ are logically contradictory. Moreover, 
by Claim 1.3, for all $\alpha, \beta \in \mathbf{G L}$, if $\bullet=$ blank, $\square, \diamond$ and $*=$ blank, $\diamond, \square$, respectively, then formulas in the following pairs are logically contradictory: $\left\{A^{\bullet} \alpha \beta, \mathrm{O}^{*} \alpha \beta\right\}$ and $\left\{\mathrm{E}^{\bullet} \alpha \beta, \mathrm{I}^{*} \alpha \beta\right\}$. These are the only pairs of the syllogistic formulas which are logically contradictory.

\subsection{Compactness of Entailment in Pure Modal Logic of Names}

We can prove that the relation $\models$ is compact just by embedding it into the consequence relation of Classical Quantified Logic (CQL), which is compact. To do this, we use a transformation of formulas from For into a set of formulas of a monadic first order language and then we will use the compactness property of CQL.

A transformation For into some monadic first-order language. For For we use a monadic first-order language $\mathcal{L}$ that has the set of variables Var := $\left\{x_{0}, x_{1}, x_{2}, \ldots\right\}$ (for ' $x_{0}$ ' we will use ' $x$ ') and the set of monadic predicates $\mathbf{P}_{\mathcal{L}}:=\left\{\alpha^{\bullet}: \alpha \in \mathbf{G L}\right.$ and $\bullet$ is either blank, or $\square$, or $\left.\nabla\right\}$. Moreover, $\mathcal{L}$ does not have any other non-logical constants. Finally, let For $_{\mathcal{L}}$ be the set of all formulas of $\mathcal{L}$ which are obtained in the standard way.

We use the following transformation $\mathbf{t}$ from For into For $_{\mathcal{L}}$, where for all $\alpha, \beta \in \mathbf{G L}, \varphi, \psi \in$ For, and $\bullet \in\{$ blank, $\square, \diamond\}$ we put:

$$
\begin{aligned}
\mathbf{t}\left(A^{\bullet} \alpha \beta\right):= & \left\ulcorner\forall_{x}\left(\alpha x \supset \beta^{\bullet} x\right)\right\urcorner, \\
\mathbf{t}(\mathrm{E} \bullet \alpha \beta):= & \left\ulcorner\neg \exists_{x}\left(\alpha x \wedge \beta^{*} x\right)\right\urcorner, \\
& \text { where } *=\text { blank, } \diamond, \square, \text { for } \bullet=\text { blank, } \square, \diamond, \text { respectively, } \\
\mathbf{t}\left(\mathrm{I}^{\bullet} \alpha \beta\right):= & \left\ulcorner\exists_{x}\left(\alpha x \wedge \beta^{\bullet} x\right)\right\urcorner, \\
\mathbf{t}\left(\mathrm{O}^{\bullet} \alpha \beta\right):= & \left\ulcorner\exists_{x}\left(\alpha x \wedge \neg \beta^{*} x\right)\right\urcorner, \\
& \text { where } *=\text { blank, } \diamond, \square, \text { for } \bullet=\text { blank, } \square, \diamond, \text { respectively, } \\
\mathbf{t}(\neg \varphi)= & \ulcorner\neg \mathbf{t}(\varphi)\urcorner, \\
\mathbf{t}(\varphi \circ \psi)= & \ulcorner\mathbf{t}(\varphi) \circ \mathbf{t}(\psi)\urcorner, \quad \text { for } \circ \in\{\wedge, \vee, \supset, \equiv\} .
\end{aligned}
$$

Moreover, for any $\Psi \subseteq$ For we put $\mathbf{t}(\Psi):=\{\mathbf{t}(\psi): \psi \in \Psi\}$. As we can see the transformation is an injection.

Models for $\mathcal{L}$. A model for $\mathcal{L}$ is any pair $\mathfrak{M}_{\mathcal{L}}=\langle D, \mathfrak{I}\rangle$, where $D$ is a nonempty set and $\mathfrak{I}$ is a function from $\mathbf{P}_{\mathcal{L}}$ into $\mathcal{P}(D)$. Let $\operatorname{Mod}_{\mathcal{L}}$ be the class of all models of $\mathcal{L}$. For any model $\mathfrak{M}_{\mathcal{L}}=\langle D, \mathfrak{I}\rangle$, any function $v$ : Var $\rightarrow D$ is a valuation of variables. We introduce, in the standard way, the notion of truth of formulas of $\mathbf{F o r}_{\mathcal{L}}$ in $\mathfrak{M}_{\mathcal{L}}$ (we write: $\mathfrak{M}_{\mathcal{L}} \vDash \varphi$ ). Of course, for all $\pi \in \mathbf{P}_{\mathcal{L}}$ and $x_{i} \in$ Var: $\mathfrak{M}_{\mathcal{L}} \vDash \pi x_{i}$ iff $v\left(x_{i}\right) \in \mathfrak{I}(\pi)$. 
For all $\Psi \in \mathcal{P}\left(\right.$ For $\left._{\mathcal{L}}\right)$ and $\varphi \in$ For $_{\mathcal{L}}$, also as usual, we say that in CQL $\Psi$ logically entails $\varphi$ (we write: $\Psi \models$ CQL $\varphi$ ) iff there is no model $\mathfrak{M}_{\mathcal{L}} \in \operatorname{Mod}_{\mathcal{L}}$ such that $\mathfrak{M}_{\mathcal{L}} \vDash \Psi$ and $\mathfrak{M}_{\mathcal{L}} \not \models \varphi$.

For any $\alpha \in \mathbf{G L}$ we consider the following sentences from For $_{\mathcal{L}}$ :

$$
\begin{aligned}
& \mathrm{T}_{\alpha}^{\square}:=\forall_{x}\left(\alpha^{\square} x \supset \alpha x\right) \\
& \mathrm{T}_{\alpha}^{\diamond}:=\forall_{x}\left(\alpha x \supset \alpha^{\diamond} x\right) .
\end{aligned}
$$

Moreover, for any $\Phi \in \mathcal{P}($ For $)$ we put:

$$
\mathrm{T}_{\Phi}:=\left\{\mathrm{T}_{\alpha}^{\square} \in \mathbf{F o r}_{\mathcal{L}}: \alpha \in \mathbf{G L}(\Phi)\right\} \cup\left\{\mathrm{T}_{\alpha}^{\diamond} \in \text { For }_{\mathcal{L}}: \alpha \in \mathbf{G L}(\Phi)\right\} .
$$

That is, $\mathrm{T}_{\Phi} \in \mathcal{P}\left(\right.$ For $\left._{\mathcal{L}}\right)$ and it is the set of all formulas of the form $\mathrm{T}_{\alpha}^{\square}$ or $\mathrm{T}_{\alpha}^{\diamond}$ in which $\alpha \in \mathbf{G} \mathbf{L}(\Phi)$. We obtain:

Lemma 1.4. For all $\Psi \in \mathcal{P}($ For $)$ and $\varphi \in$ For:

$$
\Psi \models \varphi \quad \text { iff } \quad \mathrm{T}_{\Psi \cup\{\varphi\}} \cup \mathbf{t}(\Psi) \models_{\mathrm{CQL}} \mathbf{t}(\varphi) .
$$

Proof. " $\Rightarrow$ " Let $\Psi \models \varphi, \mathfrak{M}_{\mathcal{L}}=\langle D, \mathfrak{I}\rangle \in \operatorname{Mod}_{\mathcal{L}}$, and $\mathfrak{M}_{\mathcal{L}} \vDash \mathrm{T}_{\Psi \cup\{\varphi\}} \cup \mathbf{t}(\Psi)$. Then, firstly, we have:

$(\star \star) \quad \Im\left(\alpha^{\square}\right) \subseteq \mathfrak{I}(\alpha) \subseteq \mathfrak{I}\left(\alpha^{\diamond}\right)$, for any $\alpha \in \mathbf{G L}(\Psi \cup\{\varphi\})$.

Second, we consider the following model $\mathfrak{M}=\left\langle D, d, d^{\square}, d^{\square}\right\rangle$ from Mod $\backslash$ $\left\{\mathfrak{M}_{\emptyset}\right\}$, where for all $\alpha \in \mathbf{G L}$ and $\bullet \in\{$ blank, $\square, \diamond\}$ we put:

$$
d^{\bullet}(\alpha):= \begin{cases}\Im\left(\alpha^{\bullet}\right) & \text { if } \alpha \in \mathbf{G L}(\Psi \cup\{\varphi\}) \\ \emptyset & \text { otherwise }\end{cases}
$$

Of course, for any $\chi \in \Psi \cup\{\varphi\}$ we have $(*): \mathfrak{M} \vDash \chi$ iff $\mathfrak{M}_{\mathcal{L}} \vDash \mathbf{t}(\chi)$. Hence $\mathfrak{M} \vDash \Psi$, since $\mathfrak{M}_{\mathcal{L}} \vDash \mathbf{t}(\Psi)$. Therefore $\mathfrak{M} \vDash \varphi$, by the first assumption. So $\mathfrak{M}_{\mathcal{L}} \vDash \mathbf{t}(\varphi)$, by $(*)$.

"६" Let $\mathrm{T}_{\Psi \cup\{\varphi\}} \cup \mathbf{t}(\Psi) \models_{\mathrm{CQL}} \mathbf{t}(\varphi), \mathfrak{M}=\left\langle D, d, d^{\square}, d^{\square}\right\rangle \in \operatorname{Mod} \backslash\left\{\mathfrak{M}_{\emptyset}\right\}$, and $\mathfrak{M} \vDash \Psi$. For all $\alpha \in \mathbf{G L}$ and $\bullet \in\{$ blank, $\square, \diamond\}$ we put $\mathfrak{I}\left(\alpha^{\bullet}\right):=d^{\bullet}(\alpha)$. Then $\mathfrak{M}_{\mathcal{L}}:=\langle D, \mathfrak{I}\rangle$ belongs to $\operatorname{Mod}_{\mathcal{L}}$ and satisfies the condition $(\star \star)$ for any $\alpha \in \mathbf{G L}$. Moreover, for any $\psi \in$ For we have $(*): \mathfrak{M} \vDash \psi$ iff $\mathfrak{M}_{\mathcal{L}} \vDash \mathbf{t}(\psi)$. So, by $(\star \star), \mathfrak{M}_{\mathcal{L}} \vDash \mathrm{T}_{\Psi \cup\{\varphi\}} \cup \mathbf{t}(\Psi)$, since $\mathfrak{M} \vDash \Psi$. Hence $\mathfrak{M}_{\mathcal{L}} \vDash \mathbf{t}(\varphi)$, by the first assumption. Therefore $\mathfrak{M} \vDash \varphi$, by $(*)$.

Theorem 1.5 (Compactness). For all $\Psi \in \mathcal{P}$ (For) and $\varphi \in$ For:

$$
\Psi=\varphi \quad \text { iff } \quad \text { there is } \Psi_{\text {fin }} \in \mathcal{P}_{\text {fin }}(\Psi) \text { such that } \Psi_{\text {fin }}=\varphi \text {. }
$$

Proof. " $\Rightarrow$ " Let $\Psi=\varphi$. Then $\mathrm{T}_{\Psi \cup\{\varphi\}} \cup \mathbf{t}(\Psi) \models_{\mathrm{CQL}} \mathbf{t}(\varphi)$, by Lemma 1.4. Hence, by the compactness of CQL, we obtain two finite sets $\mathrm{T}_{\Psi \cup\{\varphi\}}^{\mathrm{fin}}$ and 
$\mathbf{t}(\Psi)_{\text {fin }}$ such that $\mathrm{T}_{\Psi \cup\{\varphi\}}^{\mathrm{fin}} \cup \mathbf{t}(\Psi)_{\text {fin }} \models_{\mathrm{CQL}} \mathbf{t}(\varphi)$. Moreover, since $\mathbf{t}$ is an injection, so for some finite subset $\Psi_{\text {fin }}$ of $\Psi$ we have: $\mathbf{t}(\Psi)_{\text {fin }}=\mathbf{t}\left(\Psi_{\text {fin }}\right)$. Hence $\Psi_{\text {fin }}=\varphi$, by Lemma 1.4 .

\section{Auxiliary Singular Formulas for Tableaus}

Auxiliary singular names. Let AI be the infinite, countable set of the following auxiliary indexes: $i_{0}, i_{1}, i_{2}, \ldots$ (for the first two of these indexes we will use ' $i$ ' and ' $j$ ', respectively). These indexes are auxiliary singular names that are needed for the construction of tableaus.

Auxiliary singular formulas and their negations. An auxiliary singular formula is any expression which has one of the following forms: $\ulcorner\imath \varepsilon \alpha\urcorner,\ulcorner\imath \varepsilon \square \alpha\urcorner$, and $\left\ulcorner\imath \varepsilon^{\diamond} \alpha\right\urcorner$, for some $i \in \mathbf{A I}$ and $\alpha \in \mathbf{G L} .{ }^{6}$ We read, respectively, the auxiliary singular formulas ' $i \varepsilon a$ ', ' $i \varepsilon^{\square} a$ ', and ' $i \varepsilon^{\diamond} a$ ' as follows: " $i$ is $a$ ", " $i$ is necessarily $a$ ", and " $i$ is possibly $a$ ".

If $\sigma$ is an auxiliary singular formula, then $\ulcorner\neg \sigma\urcorner$ is its negation. Let $\boldsymbol{\Sigma}$ be the set of all auxiliary singular formulas and their negations. Moreover, let $\boldsymbol{\Sigma}\urcorner$ be the set of all negations of singular formulas; so $\boldsymbol{\Sigma}\urcorner \boldsymbol{\Sigma}$.

Notion of truth for the set For $\cup \boldsymbol{\Sigma}$. By Claim 1.2, the empty model $\mathfrak{M}_{\emptyset}$ does not have an influence on the relation $\models$. Thus, we will use only non-empty models from Mod. Moreover, for any non-empty domain $D$ we introduce a function $v: \mathbf{A I} \rightarrow D$ which is a valuation of indexes. A valuation of indexes in a model is a valuation into its domain.

For any non-empty model $\mathfrak{M}=\left\langle D, d, d^{\square}, d^{\diamond}\right\rangle$ and any valuation of indexes $v: \mathbf{A I} \rightarrow D$ we can extend the notion of truth to the set For $\cup \boldsymbol{\Sigma}$. For all $\imath \in \mathbf{A I}, \alpha \in \mathbf{G L}, \sigma \in \mathbf{\Sigma}$, and $\varphi \in$ For we put:

$\mathfrak{M}, v \vDash \imath \varepsilon^{\bullet} \alpha$ iff $v(\imath) \in d^{\bullet}(\alpha)$, for any $\bullet \in\{$ blank, $\square, \diamond\}$,

$\mathfrak{M}, v \vDash \neg \sigma$ iff $\mathfrak{M}, v \not \models \sigma$,

$\mathfrak{M}, v \vDash \varphi$ iff $\mathfrak{M} \vDash \varphi$.

Logical entailment for For $\cup \boldsymbol{\Sigma}$. For all $\Psi \in \mathcal{P}($ For $\cup \boldsymbol{\Sigma})$ and $\varphi \in$ For $\cup \boldsymbol{\Sigma}$, we say that $\Psi$ logically entails $\varphi$ (we write: $\Psi \models \varphi$ ) iff for any non-empty model $\mathfrak{M}$ and any valuation $v$ in its domain: if $\mathfrak{M}, v \vDash \Psi$, then $\mathfrak{M}, v \vDash \varphi$.

\footnotetext{
${ }^{6}$ Although we use symbol $\varepsilon$, of course it is not Stanisław Leśniewski's constant.
} 


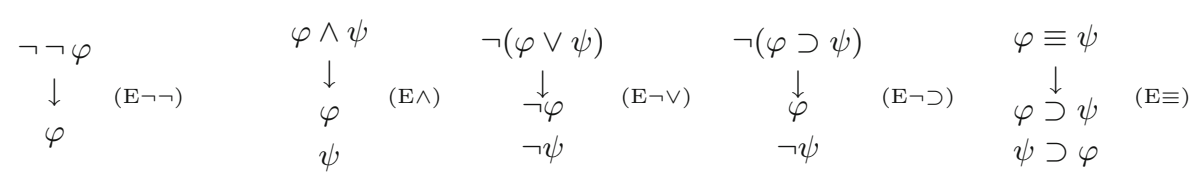

Table 1. The rule $(\mathrm{E} \neg \neg)$ and non-branching elimination rules (NBE)

Claim 2.1. For all $\alpha, \beta \in \mathbf{G L}, \imath \in \mathbf{A I}$, and $\bullet \in\{$ blank, $\square, \diamond\}$ :

(i) $A^{\bullet} \alpha \beta, \imath \varepsilon \alpha \models \imath \varepsilon^{\bullet} \beta$ and $E^{\bullet} \alpha \beta, \imath \varepsilon \alpha=\neg \imath \varepsilon^{*} \beta$, where $*=$ blank, $\diamond$, $\square$, for $\bullet=$ blank $, \square, \diamond$, respectively.

(ii) $\imath \varepsilon^{\square} \alpha=\imath \varepsilon \alpha \models \imath \varepsilon^{\diamond} \alpha$.

\section{Tableaus}

To examine $\Psi \models \varphi$ we will apply tableaus that are a kind of finite trees constructed from formulas of $\Psi \cup\{\neg \varphi\} \cup \Sigma$ by rules we introduce below.

\subsection{Tableau Rules}

Tableaus are build by so-called tableau rules. We will first outline the rules. Afterwards, we will provide a precise definition of a tableau that will formally specify their behaviour. We have seven groups of rules. Only the first three kinds decompose sentences with classical propositional connectives.

The first group consists of a single rule $(E \neg \neg)$ of elimination of double negation. Formally, this rule is the set $\{\langle\neg \neg \varphi, \varphi\rangle: \varphi \in$ For $\}$ (cf. Table 1).

The second group has four members $(\mathrm{E} \wedge), \mathrm{E} \neg \vee),(\mathrm{E} \neg \supset),(\mathrm{E} \equiv)$ and they do not lead to branches in tableaus either. We will denote these rules by (NBE) for "non-branching elimination". Formally, each rule in (NBE) is a set of triples of the form $\langle\varphi,\langle\psi, \chi\rangle\rangle$, where $\varphi, \psi, \chi \in$ For have a particular form given by the relevant schemes from Table 1 . The ordered pair $\langle\psi, \chi\rangle$ plays the role of a conjunction of conclusions.

The third group of rules, $(\mathrm{E} \vee),(\mathrm{E} \supset),(\mathrm{E} \neg \wedge),(\mathrm{E} \neg \equiv)$, make two branches in tableaus (cf. Table 2). We will denote these rules by (BE) for "branching elimination". Formally, each rule in (BE) is a set of ordered pairs $\left\langle\varphi,\left\{\psi_{1}\right.\right.$, $\left.\left.\psi_{2}\right\}\right\rangle$, where $\varphi, \psi_{1}, \psi_{2} \in$ For have a particular form given by the relevant schemes. In this case the set $\left\{\psi_{1}, \psi_{2}\right\}$ plays the role of alternative conclusions.

The rules of the other four types are divided into two categories: "rules of inference" and "rules of choice". 


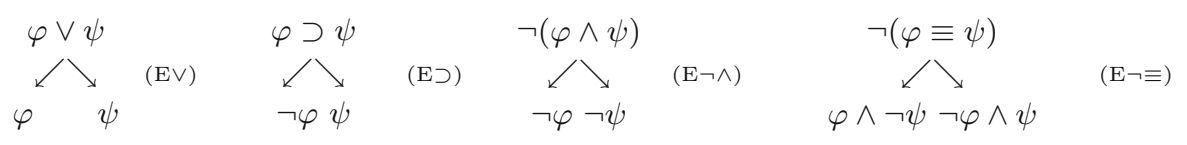

Table 2. Branching elimination rules (BE)

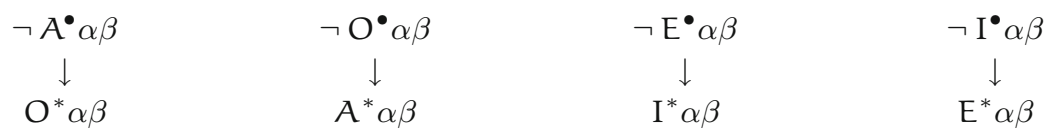

where $*=$ blank, $\diamond, \square$, for $\bullet=$ blank, $\square, \diamond$, respectively.

Table 3. Rules inference $(\mathrm{RI} \neg)$

For any $\bullet \in\{$ blank, $\square, \diamond\}:$

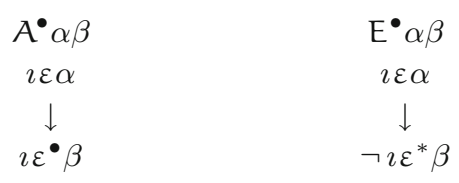

where $*=$ blank, $\diamond, \square$, for $\bullet=$ blank, $\square, \diamond$, respectively.

Table 4. Rules of inference for general formulas (RIg)

The fourth group of rules consists of twelve rules of inference; one rule for one type of categorical formulas. Each of these rules transforms the negation of a given categorical formula into a logically contradictory formula (cf. Table 3). So these twelve rules we denote by $(\mathrm{RI} \neg)$. Formally, each rule in $(\mathrm{RI} \neg)$ is a set of ordered pairs of the form $\langle\varphi, \psi\rangle$, where $\varphi, \psi \in$ For have a particular form given by the relevant schemes.

The fifth group of rules consists of six rules of inference; one rule for one type of general formulas (cf. Table 4). So these six rules we denote by (RIg). Formally, each rule in (RIg) is a set of ordered pairs of the form $\langle\{\varphi, \sigma\}, \varsigma\rangle$, where $\varphi \in$ For and $\sigma, \varsigma \in \boldsymbol{\Sigma}$ have a particular form given by the relevant schemes. In this case $\{\varphi, \sigma\}$ plays the role of a set of premises.

The sixth group of rules consists of two rules of inference for auxiliary singular formulas (cf. Table 5). So these two rules we denote by (RIa). Formally, each rule in (RIa) is a set of ordered pairs of the form $\langle\sigma, \varsigma\rangle$, where $\sigma, \varsigma \in \boldsymbol{\Sigma} \backslash \boldsymbol{\Sigma}\urcorner$ have a particular form given by the relevant schemes.

The seventh group of rules consists of six rules of choice for particular formulas; one rule for one each of particular formula (cf. Table 6). These six 


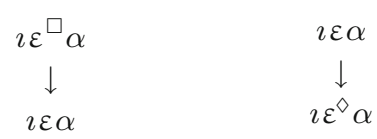

Table 5. Rules of inference for auxiliary singular formulas (RIa)

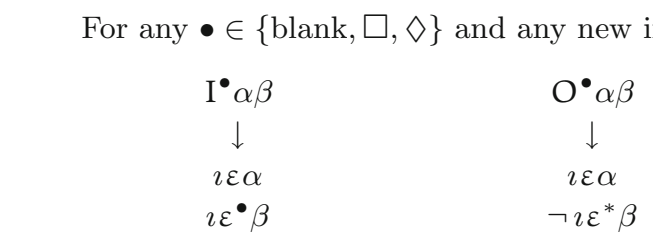

where $*=$ blank, $\diamond, \square$, for $\bullet=$ blank, $\square, \diamond$, respectively.

Table 6. Rules (RC) for particular formulas

rules we denote by (RC). Formally, each of rules of $(\mathrm{RC})$ is a triple of the form $\langle\varphi,\langle\sigma, \varsigma\rangle\rangle$, where $\varphi \in$ For and $\sigma, \varsigma \in \boldsymbol{\Sigma}$ have a specific form given by the relevant schemes. In this case $\langle\sigma, \varsigma\rangle$ plays the role of a conjunction of conclusions.

\subsection{Tableaus. Closed Tableaus. Open Tableaus}

Let $\Phi$ be a non-empty (maybe infinite) subset of For. A tableau for $\Phi$ is any NON-EMPTY FINITE TREE of formulas from For $\cup \boldsymbol{\Sigma}$ which meets all of the following four conditions:

1. The tree $T$ has only one root (i.e., the node at the top) belonging to $\Phi$.

2. From each of the nodes of $T$ at most two edges (arrows) start.

3. If from a given node in $T$ two edges start with formulas $\psi_{1}$ and $\psi_{2}$, respectively, then in the initial path from the root to this node there is a node with the formula $\varphi$ such that the pair $\left\langle\varphi,\left\{\psi_{l}, \psi_{2}\right\}\right\rangle$ belongs to some rule of $(\mathrm{BE})$.

4. For any initial path $P$ in $T$ which has the form $\left(\varphi_{1}, \ldots, \varphi_{n}\right)$ (i.e., $\varphi_{1}$ is the root of $T$ ), for any $l \leqslant n$ at least one of the following conditions is met:

(i) $\varphi_{l} \in \Phi$.

(ii) There is a $j<l$ such that $\left\langle\varphi_{j}, \varphi_{l}\right\rangle$ belongs to some rule of (E $\neg \neg$ ), or of (RIa), or of (RN).

(iii) There is a $j<l$ such that for some $\psi \in$ For the pair $\left\langle\varphi_{j},\left\{\varphi_{l}, \psi\right\}\right\rangle$ belongs to some rule of (BE) and for the initial subpath $P^{\prime}$ of $P$ which has the form $\left(\varphi_{1}, \ldots, \varphi_{j}, \ldots, \varphi_{l-1}\right)$ the path $\left(P^{\prime}, \psi\right)$ belongs to $T$. 
(iv) There are $j, k<l$ such that $\left\langle\left\{\varphi_{j}, \varphi_{k}\right\}, \varphi_{l}\right\rangle$ belongs to some rule of (RIg).

(v) There are $j<l$ and $\psi \in \mathbf{F}$ For $\cup \boldsymbol{\Sigma}$ such that for the initial subpath $P^{\prime}$ of $P$ which has the form $\left(\varphi_{1}, \ldots, \varphi_{j}, \ldots, \varphi_{l}\right)$ the path $\left(P^{\prime}, \psi\right)$ belongs to $T$ and $\left\langle\varphi_{j},\left\langle\varphi_{l}, \psi\right\rangle\right\rangle$ belongs to some rule either of (NBE) or of (RC), where in the second case the index $\imath$ from $\varphi_{l}$ and $\psi$ does not occur in the initial subpath $P^{\prime \prime}$ of $P$ which has the form $\left(\varphi_{1}, \ldots, \varphi_{l-1}\right)$

(vi) There is a $j<l-1$ such that $\left\langle\varphi_{j},\left\langle\varphi_{l-1}, \varphi_{l}\right\rangle\right\rangle$ belongs to some rule either of (NBE) or of (RC), where in the second case the index $\imath$ from $\varphi_{l-1}$ and $\varphi_{l}$ does not occur in the initial subpath $P^{\prime}$ of $P$ which has the form $\left(\varphi_{1}, \ldots, \varphi_{l-2}\right)$.

Clearly, in a tableau for some $\Phi$ only a finite subset of formulas in $\Phi$ that occur in the tableau forms a proof. So any tableau for $\Phi$ is also a tableau for each set of formulas which is a superset of $\Phi$.

Any path from a root to a bottom is called a branch. A branch in a given tableau is closed iff there are formulas from For $\cup \boldsymbol{\Sigma}$ of the form $\varphi$ and $\neg \varphi$ on two of its nodes; otherwise it is open. A tableau is closed iff every its branch is closed; otherwise is open.

\section{Tableau Proofs. Soundness}

For all $\Psi \in \mathcal{P}$ (For) and $\varphi \in$ For, we say that $\varphi$ has a tableau proof from $\Psi$ (we write: $\Psi \vdash \varphi$ ) iff there is a closed tableau for the set $\Psi \cup\{\neg \varphi\}$.

We prove that the relation $\vdash$ is sound, i.e.:

Theorem 4.1 (Soundness). For all $\Psi \in \mathcal{P}($ For $)$ and $\varphi \in$ For:

$$
\text { if } \Psi \vdash \varphi \text { then } \Psi \models \varphi \text {. }
$$

Proof. Assume that $\Psi \vdash \varphi$, i.e., there is a closed tableau $T$ for $\Psi \cup\{\neg \varphi\}$. Suppose towards a contradiction that $\Psi \not \forall \varphi$. Then there is a non-empty model $\mathfrak{M}=\left\langle D, d, d^{\square}, d^{\diamond}\right\rangle \in$ Mod such that $\mathfrak{M} \vDash \Psi$, but $\mathfrak{M} \not \models \varphi$; so $\mathfrak{M} \vDash$ $\Psi \cup\{\neg \varphi\}$.

Note that all tableau rules of the types (NBE) and (RN) preserve truth and moreover at least one conclusion derived from the application of a rule of type (BE) preserves truth. Hence for some closed branch $B$ in $T$ all formulas from For which are in $B$ are true in $\mathfrak{M}$, because $\mathfrak{M} \vDash \Psi \cup\{\neg \varphi\}$. Let For $_{B}$ be the set of all formulas from For which are in $B$. So $\mathfrak{M} \vDash$ For $_{B}$.

If in branch $B$ no rules of (RC) were used, then in $B$ there would be only formulas from For $_{B}$. But this contradicts the fact that $B$ is closed. So we 
suppose that on $B$ some rule of (RC) is used at least once. Let $\boldsymbol{\Sigma}_{B}$ be the set of all auxiliary formulas on $B$ which we obtain by rules of $(\mathrm{RC})$ and let $I_{B}$ be the set of all indexes which appear in the formulas from $\boldsymbol{\Sigma}_{B}$. We have $I_{B} \neq \emptyset$. Since rules from the group (RC) were used only to formulas from For $_{B}$ and $\mathfrak{M} \vDash$ For $_{B}$, there is a valuation $v: I_{B} \rightarrow D$ such that $\mathfrak{M}, v \vDash \boldsymbol{\Sigma}_{B}$. Since all other tableau rules preserve truth for $\mathfrak{M}$ and $v$, so by induction on the length of $B$, we show that all formulas on $B$ are also true in $\mathfrak{M}$. But this contradicts the fact that $B$ is closed.

\section{Completeness}

Since the logical consequence relation $\models$ is compact, we can focus on tableaus for non-empty finite sets. Let $\Phi$ be any non-empty finite subset of For.

In the light of Claim 1.3 we see that the rules of $(\mathrm{RC})$ do not have to be applied to all p-formulas in a given branch of a given tableau. It is enough to apply the rules to the so-called essential p-formulas in a given branch. For any tableau $T$ and any branch $B$ of $T$ a p-formula $\varphi$ is essential in $B$ iff there is no p-formula $\psi$ on $B$ such that $\psi=\varphi$ and $\varphi \not \models \psi$.

Now we introduce a special kind of tableaus, called mini-max. They are maximal since all relevant expressions are decomposed, but minimal since only relevant expressions are decomposed.

We say that a tableau $T$ for $\Phi$ is "mini-max" iff $T$ satisfies the following conditions:

1. $T$ begins with a single path in which there occur all members from $\Phi$ (we will call this path the initial list).

2. In each branch $B$ of $T$, any rule that could be applied to a formula was applied exactly once, with the exception of the following cases:

- rules of ( $\mathrm{RC}$ ) were applied only to essential p-formulas,

- rules of (RC) were applied only to one of two equivalent formulas (see points 3 and 4 of Claim 1.3),

- in $B$ we have a pair of formulas $\chi$ and $\ulcorner\neg \chi\urcorner$.

Of course, for any non-empty finite subset of For there is a "mini-max" tableau. Note that formulas of the form $\left\ulcorner\mathrm{O}^{\square} \alpha \alpha\right\urcorner$ and $\ulcorner\mathrm{O} \alpha \alpha\urcorner$ cannot appear in an open branch in "mini-max" tableaus (cf. Claim 1.3.6 and the rules of (RC) and (RIa)). All "mini-max" tableaus are complete in the standard sense [see, e.g., 13, p. 9].

In Sections 5-8 for a given branch $B$, let $p_{\mathrm{e}}^{B}$ be the number of all essential non-equivalent p-formulas in $B$. For the Completeness Theorem we use the following Completeness Lemma: 
Lemma 5.1. For any non-empty finite subset $\Phi$ of For and any "mini-max" open tableau $T$ for $\Phi$, there is a model $\mathfrak{M}_{T} \in \operatorname{Mod}$ such that $\mathfrak{M}_{T} \vDash \Phi$ and the cardinality of $\mathfrak{M}_{T}$ is equal to $\min \left\{p_{\mathrm{e}}^{B}: B\right.$ is an open branch in $\left.T\right\}$.

Proof. Let $B$ be an open branch in $T$ such that $B$ has the smallest number of essential formulas that are not equivalent to p-formulas in $B$ among all open branches in $T$. By definition of $T$, all formulas from $\Phi$ are on $B$. We construct the following model $\mathfrak{M}_{T}=\left\langle D, d, d^{\square}, d^{\diamond}\right\rangle$ from Mod. First, we put:

$$
D=\left\{\imath \in \mathbf{A I}: \exists_{\alpha \in \mathbf{G L}}\ulcorner\imath \varepsilon \alpha\urcorner \text { is in } B\right\} .
$$

Because $T$ is "mini-max", we have: $D=\emptyset$ iff there is no p-formula in $B$ iff $p_{\mathrm{e}}^{B}=0$. Moreover, $\operatorname{Card}(\boldsymbol{D})=p_{\mathrm{e}}^{B}$ and for any $\imath \in \mathbf{A I}$ we have:

$$
\imath \in D \quad \text { iff } \quad \exists_{\alpha \in \mathbf{G L}}\left\ulcorner\imath \varepsilon^{\bullet} \alpha\right\urcorner \text { is in } B \text {, for some } \bullet \in\{\text { blank, } \square, \diamond\} .
$$

Second, for all $\alpha \in \mathbf{G L}$ and $\bullet \in\{$ blank, $\square, \diamond\}$ we put:

$$
d^{\bullet}(\alpha):=\{\imath \in D:\ulcorner\imath \varepsilon \bullet \alpha\urcorner \text { is in } B\} .
$$

Now we show that $\mathfrak{M}_{T}$ is a model from Mod, i.e., for any $\alpha$ in GL: $d^{\square}(\alpha) \subseteq d(\alpha) \subseteq d^{\diamond}(\alpha)$. Indeed, if $\imath \in d^{\square}(\alpha)$, then $\left\ulcorner\imath \varepsilon^{\square} \alpha\right\urcorner$ is in $B$. Hence, by some rule of (RIa), also $\ulcorner\imath \varepsilon \alpha\urcorner$ is in $B$, since $T$ is "mini-max". So $\imath \in d(\alpha)$. Similarly we show that $d(\alpha) \subseteq d^{\diamond}(\alpha)$.

Now we prove that for any $\varphi \in$ For we obtain: if $\varphi$ is in $B$, then $\mathfrak{M}_{T} \vDash \varphi$. The proof is by induction on the complexity of formulas.

(a) We consider the case of formulas of For $_{\text {syll }}$.

Suppose that for some $\bullet \in\{$ blank, $\square, \diamond\},\left\ulcorner\mathrm{I}^{\bullet} \alpha \beta\right\urcorner$ is in $B$. Then, if $\left\ulcorner\mathrm{I}^{\bullet} \alpha \beta\right\urcorner$ is essential in $B$, then by some rule of (RC), for some $\imath \in \mathbf{A I}$ we have: $\ulcorner\imath \varepsilon \alpha\urcorner$ and $\left\ulcorner\imath \varepsilon^{\bullet} \beta\right\urcorner$ are in $B .{ }^{7}$ If $\left\ulcorner\mathrm{I}^{\bullet} \alpha \beta\right\urcorner$ is not essential in $B$ then $\bullet \neq \square$. So if $\bullet$ - blank then $\left\ulcorner\mathrm{I}^{\square} \alpha \beta\right\urcorner$ is in $B$. Hence, by some rule of (RC), for some $\imath \in \mathbf{A I}$ we have: $\ulcorner\imath \varepsilon \alpha\urcorner$ and $\ulcorner\imath \varepsilon \square \beta\urcorner$ are in $B$. So, by some rule of (RIa), $\ulcorner\imath \varepsilon \beta\urcorner$ is also in $B$. Moreover, if $\bullet=\diamond$ then $\left\ulcorner\mathrm{I}^{*} \alpha \beta\right\urcorner$ is in $B$, for some $* \in\{$ blank, $\square$ \}. Hence, by some rule of (RC), for some $\imath \in \mathbf{A I}$ we have: $\ulcorner\imath \varepsilon \alpha\urcorner$ and $\left\ulcorner\imath \varepsilon^{*} \beta\right\urcorner$ are in $B$. So, by some rule of (RIa), $\left\ulcorner\imath \varepsilon^{\diamond} \beta\right\urcorner$ is also in $B$. Thus, in all cases, $\imath \in d(\alpha) \cap d^{\bullet}(\beta) ;$ so $\mathfrak{M}_{T} \vDash \mathrm{I}^{\bullet} \alpha \beta$.

Suppose that $\left\ulcorner\mathrm{O}^{\bullet} \alpha \beta\right\urcorner$ is in $B$, for some $\bullet \in\{$ blank, $\square, \diamond\}$. Then, if $\left\ulcorner\mathrm{O}^{\bullet} \alpha \beta\right\urcorner$ is essential in $B$, then by some rule of (RC), for some $\imath \in \mathbf{A I}$ we have: $\ulcorner\imath \varepsilon \alpha\urcorner$ and $\left\ulcorner\neg \imath \varepsilon^{*} \beta\right\urcorner$ are in $B$, where $*=$ blank, $\diamond, \square$, for $\bullet=$ blank, $\square, \diamond$,

\footnotetext{
${ }^{7}$ Sometimes the effect is because some rule of (RC) was applied to a formula equivalent to $\left\ulcorner\mathrm{I}^{\bullet} \alpha \beta\right\urcorner$, since $B$ is a branch of a "mini-max". For example, $\bullet=$ blank and rule of (RC) was applied to $\ulcorner\mathrm{I} \beta \alpha\urcorner$, or $\alpha=\beta$ and $\bullet \in\{$ blank, $\diamond$ \}, but rule of (RC) was applied either to $\ulcorner\mathrm{I} \alpha \alpha\urcorner$ or to $\left\ulcorner\mathrm{I}^{\diamond} \alpha \alpha\right\urcorner$.
} 
respectively. Hence $\imath \in d(\alpha)$ and $\left\ulcorner\imath \varepsilon^{*} \beta\right\urcorner$ is not in $B$, since $B$ is open. If $\left\ulcorner\mathrm{O}^{\bullet} \alpha \beta\right\urcorner$ is not essential in $B$ then $\bullet \neq \square$. So if $\bullet=$ blank then $\left\ulcorner\mathrm{O}^{\square} \alpha \beta\right\urcorner$ is in $B$. Therefore, by some rule of (RC), for some $\imath \in \mathbf{A I}$ we have: $\ulcorner\imath \varepsilon \alpha\urcorner$ and $\left\ulcorner\neg \imath \varepsilon^{\diamond} \beta\right\urcorner$ are in $B$. Hence $\imath \in d(\alpha)$ and $\left\ulcorner\imath \varepsilon^{\diamond} \beta\right\urcorner$ is not in $B$, since $B$ is open. So, by some rule of (RIa), also $\ulcorner\imath \varepsilon \beta\urcorner$ is not in $B$. Moreover, if $\bullet=\diamond$ then $\left\ulcorner\mathrm{O}^{*} \alpha \beta\right\urcorner$ is in $B$, for some $* \in\{$ blank, $\square$. Therefore, by some rule of (RC), for some $\imath \in \mathbf{A I}$ we have: $\ulcorner\imath \varepsilon \alpha\urcorner$ and $\left\ulcorner\neg \imath \varepsilon^{\star} \beta\right\urcorner$ are in $B$, where $\star=$ blank, $\diamond$, for $*=$ blank, $\square$, respectively. Hence $\imath \in d(\alpha)$ and $\left\ulcorner\imath \varepsilon^{\star} \beta\right\urcorner$ is not in $B$, since $B$ is open. So, by some rule of (RIa), also $\left\ulcorner\imath \varepsilon^{\diamond} \beta\right\urcorner$ is not in $B$. Thus, in all cases, $\imath \notin d^{*}(\beta)$, where $*=$ blank, $\diamond, \square$, for $\bullet=$ blank, $\square, \diamond$, respectively. Therefore $d(\alpha) \nsubseteq d^{*}(\beta)$, i.e., $\mathfrak{M}_{T} \vDash \mathrm{O}^{\bullet} \alpha \beta$.

Suppose that $\left\ulcorner A^{\bullet} \alpha \beta\right\urcorner$ is in $B$, for some $\bullet \in\{$ blank, $\square, \diamond\}$. If $\imath \in d(\alpha)$ then $\ulcorner\imath \varepsilon \alpha\urcorner$ is in $B$. So also $\left\ulcorner\imath \varepsilon^{\bullet} \beta\right\urcorner$ is in $B$, by some rule of (RIg). Hence $\imath \in d^{\bullet}(\beta)$. Thus, $d(\alpha) \subseteq d^{\bullet}(\beta)$, i.e., $\mathfrak{M}_{T} \vDash A^{\bullet} \alpha \beta$.

Suppose that $\ulcorner\mathrm{E} \bullet \alpha \beta\urcorner$ is in $B$, for some $\bullet \in\{$ blank, $\square, \diamond\}$. If $\imath \in d(\alpha)$ then $\ulcorner\imath \varepsilon \alpha\urcorner$ is in $B$. Hence, by some rule of (RIg), $\left\ulcorner\neg \imath \varepsilon^{*} \beta\right\urcorner$ is in $B$, where $*=$ blank, $\diamond, \square$, for $\bullet=$ blank, $\square, \diamond$, respectively. So $\left\ulcorner\imath \varepsilon^{*} \beta\right\urcorner$ is not in $B$, since $B$ is open. Hence $\imath \notin d^{*}(\beta)$. Thus, $d(\alpha) \cap d^{*}(\beta)=\emptyset$, i.e., $\mathfrak{M}_{T} \vDash \mathrm{E} \bullet \alpha \beta$.

(b) Now we consider the case of negation of formulas from $\mathbf{F o r}_{\text {syll }}$. Suppose that $\left\ulcorner\neg \mathrm{I}^{\bullet} \alpha \beta\right\urcorner$ (resp. $\left\ulcorner\neg \mathrm{O}^{\bullet} \alpha \beta\right\urcorner,\left\ulcorner\neg \mathrm{A}^{\bullet} \alpha \beta\right\urcorner,\left\ulcorner\neg \mathrm{E}^{\bullet} \alpha \beta\right\urcorner$ ) is in $B$, for some $\bullet \in$ $\{$ blank, $\square, \diamond\}$. Then, by some rule of $(\mathrm{RN}),\left\ulcorner\mathrm{E}^{*} \alpha \beta\right\urcorner$ (resp. $\left\ulcorner\mathrm{A}^{*} \alpha \beta\right\urcorner,\left\ulcorner\mathrm{O}^{*} \alpha \beta\right\urcorner$, $\left.\left\ulcorner\mathrm{I}^{*} \alpha \beta\right\urcorner\right)$ is in $B$, where $*=$ blank, $\diamond, \square$, for $\bullet=$ blank, $\square, \diamond$, respectively. So, by (a), $\mathfrak{M}_{T} \vDash E^{*} \alpha \beta$ (resp. $\mathfrak{M}_{T} \vDash A^{*} \alpha \beta, \mathfrak{M}_{T} \vDash \mathrm{O}^{*} \alpha \beta, \mathfrak{M}_{T} \vDash \mathrm{I}^{*} \alpha \beta$ ). Hence $\mathfrak{M}_{T} \vDash \neg I^{\bullet} \alpha \beta$ (resp. $\mathfrak{M}_{T} \vDash \neg \mathrm{O}^{\bullet} \alpha \beta, \mathfrak{M}_{T} \vDash \neg \mathrm{A}^{\bullet} \alpha \beta, \mathfrak{M}_{T} \vDash \neg \mathrm{E} \bullet \alpha \beta$ ).

(c) For other complex formulas we naturally consider inductive steps. We use the elimination rules and the fact that $T$ is "mini-max".

Since all formulas from $\Phi$ are in $B$, so $\mathfrak{M}_{T} \vDash \Phi$.

By Lemma 5.1 and Theorem 1.5 (compactness of the relation $=$ ) we obtain that the tableau approach is complete.

Theorem 5.2 (Completeness). For all $\Psi \subseteq$ For and $\varphi \in$ For:

$$
\text { if } \Psi \models \varphi \text {, then } \Psi \vdash \varphi \text {. }
$$

Proof. Let $\Psi=\varphi$. Then, by the compactness of $\models$, there is a finite subset $\Psi_{\text {fin }}$ of $\Psi$ such that $\Psi_{\text {fin }}=\varphi$. Now we suppose towards contradiction that $\Psi_{\text {fin }} \nvdash \varphi$. Then there is a "mini-max" open tableau $T$ for $\Psi_{\text {fin }} \cup\{\neg \varphi\}$. Hence, by Lemma 5.1, there is a model $\mathfrak{M}_{T} \in$ Mod such that $\mathfrak{M}_{T} \vDash \Psi_{\text {fin }} \cup\{\neg \varphi\}$. So we obtain a contradiction: $\Psi_{\text {fin }} \not \models \varphi$. Thus, $\Psi_{\text {fin }} \vdash \varphi$. So also $\Psi \vdash \varphi$. 


\section{Decidability and Cardinalities of Models}

The decidability of $\Psi \models \varphi$ for the pure modal logic of names is considered in the case when $\Psi$ is finite. Thus, let $\Psi \in \mathcal{P}_{\text {fin }}($ For $)$. The decidability of $\Psi \models \varphi$ can be tested in the following two ways.

First, we have already established decidability on the basis of Lemma 1.4, since the relation $\models_{\mathrm{CQL}}$ is decidable. However, we dare for more! We want to estimate the cardinalities of models for the logic under research that are less than the cardinalities of models for CQL. It is known that, for $\Psi \in \mathcal{P}\left(\right.$ For $\left._{\mathcal{L}}\right)$ and $\varphi \in$ For $_{\mathcal{L}}$, in order to check whether $\Psi \models$ CQL $\varphi$ we have to examine models with a cardinality not bigger than $2^{k}$, where $k$ is the number of monadic predicates of $\mathbf{P}_{\mathcal{L}}$ that occur in the formulas from the set $\Psi \cup\{\varphi\}$. Let us note that we mean not only letters from $\mathbf{G L}$, but also all instances of the form $\alpha \bullet$, where $\alpha \in \mathbf{G L}$ and $\bullet \in\{$ blank, $\square, \diamond\}$.

Either by Lemma 1.4 and the decidability of monadic first-order logic, or by Lemma 5.1 and Theorem 4.1 we obtain:

Theorem 6.1 (Decidability). For all $\Psi \in \mathcal{P}_{\text {fin }}($ For $)$ and $\varphi \in$ For, the problem whether $\Psi=\varphi$ is decidable.

Proof. For $\Psi \cup\{\neg \varphi\}$ we build some "mini-max" tableau. If the tableau is closed, then $\Psi \vdash \varphi$. So $\Psi \models \varphi$, by soundness (Theorem 4.1). If the tableau is open, then $\Psi \not \forall \varphi$, by Lemma 5.1.

As we declared, we would like to estimate the cardinality of a given model to check if $\Psi=\varphi .^{8}$ First, we obtain the following theorem for formulas that have a disjunctive normal form. Note that each formula $\chi$ from For can be written in a disjunctive normal form $\chi^{\mathrm{d}}$. Moreover, let $\Psi^{\mathrm{d}}:=\left\{\psi^{\mathrm{d}}: \psi \in \Psi\right\}$. Theorem 6.2. For all $\Psi \in \mathcal{P}_{\text {fin }}($ For $)$ and $\varphi \in$ For the following conditions are equivalent:

(a) $\Psi \nvdash \varphi$.

(b) $\Psi^{\mathrm{d}} \nvdash \varphi^{\mathrm{d}}$.

(c) For some mini-max open tableau $T$ for $\Psi^{\mathrm{d}} \cup\left\{(\neg \varphi)^{\mathrm{d}}\right\}$ there is a model $\mathfrak{M}_{T} \in$ Mod such that $\mathfrak{M}_{T} \vDash \Psi^{\mathrm{d}} \cup\left\{(\neg \varphi)^{\mathrm{d}}\right\}$ and the cardinality of $\mathfrak{M}_{T}$ is equal to $\min \left\{p_{\mathrm{e}}^{B}: B\right.$ is an open branch in $\left.T\right\}$.

(d) $\Psi^{\mathrm{d}} \not \models \varphi^{\mathrm{d}}$.

\footnotetext{
${ }^{8}$ In [3], we carried out a similar examination for modal syllogisms. There, we also gave the length of tableaus for modal syllogisms. But those tableaus were not branching. Here, we are not concerned with the length of tableaus.
} 
(e) $\Psi \not \forall \varphi$.

Proof. "(a) $\Leftrightarrow(\mathrm{e})$ " and " (b) $\Leftrightarrow(\mathrm{d})$ " By Theorems 4.1 and 5.2.

"(b) $\Rightarrow(\mathrm{c})$ " Let $\Psi^{\mathrm{d}} \nvdash \varphi^{\mathrm{d}}$. Then all tableaus for $\Psi^{\mathrm{d}} \cup\left\{(\neg \varphi)^{\mathrm{d}}\right\}$ are open and there exists a "mini-max" open tableau for $\Psi^{\mathrm{d}} \cup\left\{(\neg \varphi)^{\mathrm{d}}\right\}$. So we use Lemma 5.1. "(c) $\Rightarrow(\mathrm{d})$ " By definition. "(d) $\Leftrightarrow(\mathrm{e})$ " This case is obvious.

Now we assume the following notations. For any formula $\chi$, let $k_{\chi}$ be the number of conjunctions occurring in $\chi^{\mathrm{d}}$. For any $i$ from $\left\{1, \ldots, k_{\chi}\right\}$, let $\mathrm{C}_{\chi}^{i}$ be the set of all non-negated $\mathrm{p}$-formulas and all negated g-formulas that occur in the $i$-th conjunction in $\chi^{\mathrm{d}} \cdot 9$

We put the members of $\Psi$ as a sequence $\left(\psi_{1}, \ldots, \psi_{n}\right)$ (for $\Psi=\emptyset$ we put $n:=0)$. Let $\Lambda$ be the set of all sequences $\left(l_{1}, \ldots, l_{n}, l_{n+1}\right)$ of numbers such that $1 \leqslant l_{i} \leqslant k_{\psi_{i}}$, for $i=1, \ldots, n$, and $1 \leqslant l_{n+1} \leqslant k_{\neg \varphi}$. Then for any $\lambda=\left(l_{1}, \ldots, l_{n}, l_{n+1}\right) \in \Lambda$ we define the following set:

$$
\mathrm{B}^{\lambda}:=\mathrm{C}_{\psi_{1}}^{l_{1}} \cup \cdots \cup \mathrm{C}_{\psi_{n}}^{l_{n}} \cup \mathrm{C}_{\neg \varphi}^{l_{n+1}} .
$$

Clearly, any set $\mathrm{B}^{\lambda}$, for $\lambda \in \Lambda$, corresponds to a branch in a tableau build from $\Psi^{\mathrm{d}} \cup\left\{(\neg \varphi)^{\mathrm{d}}\right\}$. For any $\lambda \in \Lambda$ we define the essential p-formulas in the set $\mathrm{B}^{\lambda}$, as for a given branch of a given tableau (see p. 13). Let $p_{\mathrm{e}}^{\mathrm{B}^{\lambda}}$ be the number of all essential, non-equivalent p-formulas in $\mathrm{B}^{\lambda}$.

Once again let us note that formulas having one of the forms $\left\ulcorner\mathrm{O}^{\square} \alpha \alpha\right\urcorner$, $\ulcorner\mathrm{O} \alpha \alpha\urcorner,\left\ulcorner\neg \mathrm{A}^{\diamond} \alpha \alpha\right\urcorner$, and $\ulcorner\neg \mathrm{A} \alpha \alpha\urcorner$ cannot appear in any open branch in "minimax" tableaus. So let $\Lambda^{*}$ be the set of all $\lambda \in \Lambda$ such that no such formula is a member of set $\mathrm{B}^{\lambda}$. We put $\operatorname{Max}(\Psi, \varphi):=\max \left\{p_{\mathrm{e}}^{\mathrm{B}^{\lambda}}: \lambda \in \Lambda^{*}\right\} \cdot{ }^{10}$ From Theorem 6.2 we obtain:

Theorem 6.3. For all $\Psi \in \mathcal{P}_{\text {fin }}($ For $)$ and $\varphi \in$ For:

$\Psi \models \varphi$ iff there is no $\mathfrak{M} \in$ Mod such that $\mathfrak{M} \vDash \Psi \cup\{\neg \varphi\}$ and the power of $\mathfrak{M}$ is not greater than $\operatorname{Max}(\Psi, \varphi)$.

Proof. " $\Rightarrow$ " Obvious. " $\Leftarrow "$ Suppose that $\Psi \not \models \varphi$. Then, by Theorem 6.2, for some mini-max open tableau $T$ for $\Psi^{\mathrm{d}} \cup\left\{(\neg \varphi)^{\mathrm{d}}\right\}$ there is a model $\mathfrak{M}_{T}$ from Mod such that $\mathfrak{M}_{T} \vDash \Psi^{\mathrm{d}} \cup\left\{(\neg \varphi)^{\mathrm{d}}\right\}$ and the cardinality of $\mathfrak{M}_{T}$ is equal to $\min \left\{p_{\mathrm{e}}^{B}: B\right.$ is an open branch in $\left.T\right\}$. Hence the cardinality of $\mathfrak{M}_{T}$ is not greater than $\operatorname{Max}(\Psi, \varphi)$. Of course, $\mathfrak{M}_{T} \vDash \Psi \cup\{\neg \varphi\}$, too.

\footnotetext{
${ }^{9}$ We apply of course rules from (RN) to negated g-formulas and receive corresponding non-negated p-formulas.

${ }^{10}$ Note that $\operatorname{Max}(\Psi, \varphi)$ does not depend on the enumerated members of set $\Psi$ and moreover $\operatorname{Max}(\Psi, \varphi)=\operatorname{Max}(\emptyset, \bigwedge \Psi \rightarrow \varphi)$.
} 
Let $\boldsymbol{S}=\langle\Psi, \varphi\rangle$ be such a syllogism, where $\Psi \in \mathcal{P}_{\text {fin }}\left(\right.$ For $\left._{\text {syll }}\right) \backslash\{\emptyset\}$ and $\varphi \in$ For $_{\text {syll }}$ (see p. 3). Then the set $\Lambda$ has one element and any tableau for $\Psi \cup\{\neg \varphi\}$ has got only one branch. Of course, if either some formula of the form $\ulcorner\mathrm{O} \square \alpha \alpha\urcorner$ or $\ulcorner\mathrm{O} \alpha \alpha\urcorner$ belongs to $\Psi$, or $\varphi$ has the form $\left\ulcorner\mathrm{A}^{\diamond} \alpha \alpha\right\urcorner$ or $\ulcorner\mathrm{A} \alpha \alpha\urcorner$, then $\Psi=\varphi$. In the other case we obtain that an upper estimation of the cardinality of models is the number $p_{\mathrm{e}}^{S}$ of essential, but non-equivalent pformulas in $\Psi \cup\{\bar{\varphi}\}$, where $\bar{\varphi}$ is the formula of $\mathbf{F o r}_{\text {syll }}$ which we obtain from $\ulcorner\neg \varphi\urcorner$, by the suitable rule of (RN).

Of course, if $\boldsymbol{S}=\langle\Psi, \varphi\rangle$ is non-modal then, in the second case, $p_{\mathrm{e}}^{S}$ is the number of non-modal particular non-equivalent formulas in the set $\Psi \cup\{\bar{\varphi}\}$. In $[2,3]$ some larger estimations were made. An upper estimation of the cardinality of models was the number of non-modal particular formulas in $\Psi \cup\{\bar{\varphi}\}$. However, in those papers two various approaches to the presented problem were outlined.

In $[9,11]$ the cardinalities of models for non-modal pure logic of names were also examined. For any non-modal formula $\varphi$ from For the upper estimation of cardinalities of its models is $\frac{1}{2} n(n+1)$, where $n$ is the number of letters from GL occurring in $\varphi$. It is difficult to compare that number with number $\operatorname{Max}(\emptyset, \varphi)$ as it does not make sense to try to count the number of non-modal particular non-equivalent formulas built with letters occuring in $\varphi$.

Moreover, even for the non-modal pure logic of names, it would be a mistake to compare our results with the results presented by Kulicki $[5,6]$. First, Kulicki used a so-called strong interpretation for formulas of the form $\ulcorner A \alpha \beta\urcorner: \mathfrak{M} \vDash A \alpha \beta$ iff $\emptyset \neq d(\alpha) \subseteq d(\beta)$. So we have: $A \alpha \beta \models \mathrm{I} \alpha \beta$. Second, Kulicki examined the cardinalities of models only for Horn formulas which have the form $\left\ulcorner\left(\chi_{1} \wedge \cdots \wedge \chi_{n}\right) \rightarrow \chi_{n+1}\right\urcorner$, where $\chi_{i}$ is $\ulcorner\mathrm{A} \alpha \beta\urcorner$ or $\left\ulcorner\mathrm{I} \alpha^{\prime} \beta^{\prime}\right\urcorner$, for some $\alpha, \beta, \alpha^{\prime}, \beta^{\prime} \in \mathbf{G L}$. These Horn formulas correspond only to syllogisms that are limited to those two kind of categorical sentences.

\section{Tableau Proofs with Other Version of Tableau Contradiction}

To make tableau proofs shorter we may use an extended version of the notion of tableau contradiction and apply it to our earlier notions of the closed tableau and the tableau proof. Hence, to the pairs of tableau contradictory formulas we defined earlier we add all pairs of logically contradictory syllogistic formulas from For $_{\text {syll }}$ (see p. 5). So, a branch in a given tableau is closed, if at some of its node there is a formula either of the from $\ulcorner\mathrm{O} \alpha \alpha\urcorner$ 
$\mathrm{I} a b, \mathrm{~A} a b \vee \mathrm{E} a b \vdash \mathrm{A} a b$ (in both versions):

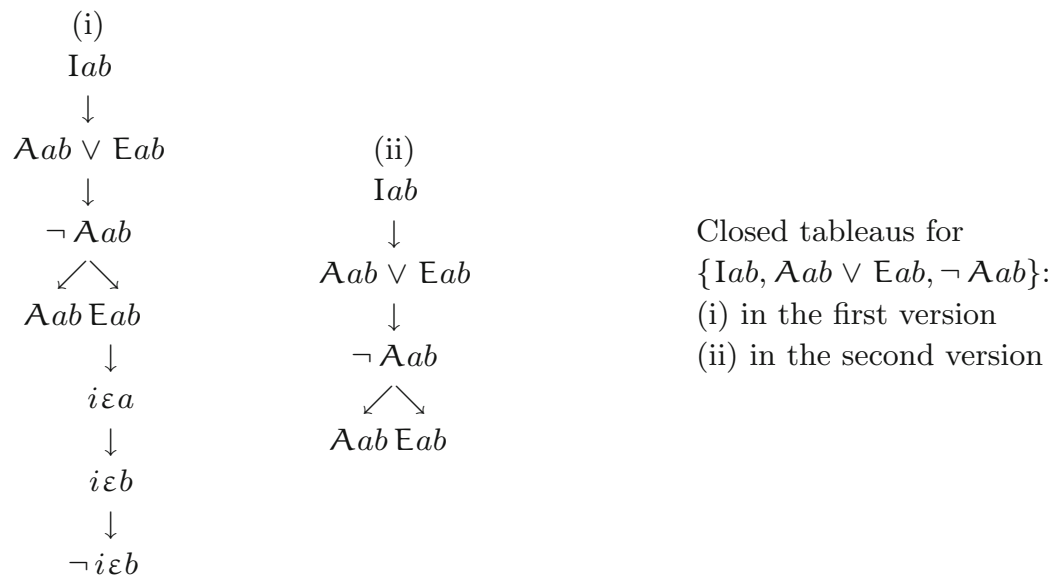

Table 7. Example of proofs with regular and extended notion of tableau contradiction.

or of the from $\left\ulcorner\mathrm{O}^{\square} \alpha \alpha\right\urcorner$ (cf. Claim 1.3.6). Due to that we have the following fact:

Proposition 7.1. $\Psi \vdash \varphi$ in the first version iff $\Psi \vdash \varphi$ in the second version.

Proof. " $\Rightarrow$ " This case is obvious.

" $\Leftarrow$ " Suppose that $\Psi \vdash \varphi$ in the second version and for $\Psi \cup\{\neg \varphi\}$ there is a closed tableau $T$ such that $T$ has a branch $B$ which is not closed in the first version of tableau contradiction. Then on $B$ we have either $\ulcorner\mathrm{O} \alpha \alpha\urcorner$, or $\left\ulcorner\mathrm{O}^{\square} \alpha \alpha\right\urcorner$, or both $\left\ulcorner A^{\bullet} \alpha \beta\right\urcorner$ and $\left\ulcorner\mathrm{O}^{*} \alpha \beta\right\urcorner$, or both $\ulcorner\mathrm{E} \bullet \alpha \beta\urcorner$ and $\left\ulcorner\mathrm{I}^{*} \alpha \beta\right\urcorner$, where $*=$ blank, $\diamond, \square$ for $\bullet=$ blank, $\square, \diamond$, respectively. Hence, by use of appropriate rules of the types (RC), (RIa), and (RIg) we obtain, in the first case: $\ulcorner\imath \varepsilon \alpha\urcorner$ and $\ulcorner\neg \imath \varepsilon \alpha\urcorner$; in the second case: $\ulcorner\imath \varepsilon \alpha\urcorner,\left\ulcorner\neg \imath \mathcal{E}^{\diamond} \alpha\right\urcorner$, and $\left\ulcorner\imath \mathcal{E}^{\diamond} \alpha\right\urcorner$; in the third case: $\ulcorner\imath \varepsilon \alpha\urcorner,\left\ulcorner\neg \imath \varepsilon^{\bullet} \beta\right\urcorner$, and $\left\ulcorner\imath \varepsilon^{\bullet} \beta\right\urcorner$; in the fourth case: $\ulcorner\imath \varepsilon \alpha\urcorner$, $\left\ulcorner\imath \varepsilon^{*} \beta\right\urcorner$, and $\left\ulcorner\neg \imath \varepsilon^{*} \beta\right\urcorner$. So we obtain a branch $B^{\prime}$ which is closed in the first version (see the example in Table 7 ).

\section{Pure Modal Logic for Both General and Singular Names}

So far we have considered the pure modal logic of names only for general names. It includes indexes for auxiliary singular names only in tableau proofs. Now-beside the letters from GL representing general names-we would like to use also schematic letters representing «real» singular names. 
We shall show that our examination of the logic of general names from the former sections can be successfully repeated for the logic of both kinds of names.

\subsection{Formulas}

We fix a countably infinite set SL which contains schematic letters: $n_{0}, n_{1}$, $n_{2}, \ldots$ (for the first two schematic letters we will use ' $n$ ' and ' $m$ ', respectively). We assume that these letters represent various singular names.

A singular formula is any expression which has one of the following forms: $\ulcorner\nu \varepsilon \alpha\urcorner,\left\ulcorner\nu \mathcal{E}^{\square} \alpha\right\urcorner$, and $\left\ulcorner\nu \mathcal{E}^{\diamond} \alpha\right\urcorner$, where $\nu \in \mathbf{S L}$ and $\alpha \in \mathbf{G L}$. Let $\mathbf{F o r}_{\text {sin }}$ be the set of all singular formulas.

Now, formulas are made of formulas $\mathbf{F o r}_{\text {syll }}$ and $\mathbf{F o r}_{\text {sin }}$ by the use of Boolean connectives and brackets. Let For ${ }^{+}$be the smallest set such that

- For $_{\text {syll }} \cup$ For $_{\text {sin }} \subseteq$ For $^{+}$;

- if $\varphi \in$ For $^{+}$, than $\ulcorner\neg \varphi\urcorner \in$ For $^{+}$;

- if $\varphi, \psi \in$ For $^{+}$, than $\ulcorner(\varphi \circ \psi)\urcorner \in$ For $^{+}$, where $\circ \in\{\wedge, \vee, \supset, \equiv\}$.

\subsection{Semantics}

Models and the notion of truth for For ${ }^{+}$. A model for For $^{+}$we call any quintuple $\left\langle D, d^{s}, d, d^{\square}, d^{\diamond}\right\rangle$, where $D$ is a non-empty set, $d^{s}$ is a function from SL into $D$, and the quadruple $\left\langle D, d, d^{\square}, d^{\diamond}\right\rangle$ is a non-empty model from Mod for For (see p. 4). Let $\mathrm{Mod}^{+}$be the class of all models for For $^{+}$.

The notion of truth for $\mathbf{F o r}_{\text {sin }}$ is similar to that which we used for $\boldsymbol{\Sigma}$ (here denotation function $d^{s}$ replaces valuation $v$ ). We use the following interpretation of formulas from For $^{+}$in all models $\mathfrak{M}=\left\langle D, d^{s}, d, d^{\square}, d^{\diamond}\right\rangle \in$ $\operatorname{Mod}^{+}, \nu \in \mathbf{S L}, \alpha \in \mathbf{G L}$, and $\bullet \in\{$ blank, $\square, \diamond\}:$

$$
\mathfrak{M} \vDash \nu \varepsilon^{\bullet} \alpha \text { iff } d^{s}(\nu) \in d^{\bullet}(\alpha) .
$$

The remaining conditions have been left unchanged (the are on p. 4).

Logical entailment, equivalence, and contradiction. These three notions are introduced for For $^{+}$in the same way as for For on p. 5. We obtain:

Claim 8.1. For all $\alpha, \beta \in \mathbf{G L}$ and $\nu \in \mathbf{S L}$ :

1. $A^{\bullet} \alpha \beta, \nu \varepsilon \alpha=\nu \varepsilon^{\bullet} \beta$, for any $\bullet \in\{$ blank, $\square, \diamond\}$.

2. $\mathrm{E}^{\bullet} \alpha \beta, \nu \varepsilon \alpha=\neg \nu \varepsilon^{\bullet} \beta$, for any $\bullet \in\{$ blank, $\square, \diamond\}$.

3. $\nu \varepsilon^{\square} \alpha \models \nu \varepsilon \alpha=\nu \varepsilon^{\diamond} \alpha$.

4. $\nu \mathcal{\varepsilon} \alpha, \nu \varepsilon^{\bullet} \beta \models \mathrm{I}^{\bullet} \alpha \beta$, for any $\bullet \in\{$ blank, $\square, \diamond\}$. 
5. $\nu \varepsilon \alpha, \neg \nu \varepsilon^{\bullet} \beta \models \mathrm{O}^{*} \alpha \beta$, where $*=$ blank, $\diamond, \square$, for $\bullet=$ blank, $\square, \diamond$, respectively.

Compactness of logical entailment. We obtain this result in a way similar to how obtained it for For in Section 1.3. However, we use a monadic first-order logic with individual constants [see, e.g., 12].

Now we use the monadic first-order language $\mathcal{L}^{+}$with monadic predicates from $\mathbf{P}_{\mathcal{L}}$ and individual constants from $\mathbf{S L}$. So we have also atomic formulas of the form $\ulcorner\pi \nu\urcorner$, where $\pi \in \mathbf{P}_{\mathcal{L}}$ and $\nu \in \mathbf{S L}$. Let For $_{\mathcal{L}^{+}}$be the set of all formulas of $\mathcal{L}^{+}$which we obtain in a standard way.

The transformation $\mathbf{t}$ from p. 6 is enriched with the following condition for all $\nu \in \mathbf{S L}$ and $\alpha \in \mathbf{G L}$ :

$$
\mathbf{t}\left(\nu \varepsilon^{\bullet} \alpha\right):=\ulcorner\alpha \bullet \nu\urcorner \text {, for any } \bullet \in\{\text { blank, } \square, \diamond\} .
$$

Ordinarily, a model for $\mathcal{L}^{+}$is any pair $\mathfrak{M}_{\mathcal{L}^{+}}=\langle D, \mathfrak{I}\rangle$, where $D$ is a nonempty set and $\mathfrak{I}$ is a function from SL into $D$ and from $\mathbf{P}_{\mathcal{L}}$ into $\mathcal{P}(D)$.

We obtain the relevant counterpart of Lemma 1.4. Hence, by the compactness of Classical Quantified Logic, we also obtain the relevant version of Theorem 1.5, which is that the relation $\models$ for For $^{+}$is also compact.

Soundness, completeness and decidability. We use the same notion of tableau and the same tableau rules as in Section 3. The only difference is that now in the description of the rules a variable ' $l$ ' takes values from the union of sets AI and SL, whereas in the former case it only took values from the set AI. Using the above rules we obtain the following facts that correspond to those in Claim 8.1:

CLAim 8.2. For all $\alpha, \beta \in \mathbf{G L}$ and $\nu \in \mathbf{S L}$ :

1. $A^{\bullet} \alpha \beta, \nu \varepsilon \alpha \vdash \nu \varepsilon^{\bullet} \beta$, for any $\bullet \in\{$ blank, $\square, \diamond\}$.

2. $\mathrm{E}^{\bullet} \alpha \beta, \nu \varepsilon \alpha \vdash \neg \nu \varepsilon^{\bullet} \beta$, for any $\bullet \in\{$ blank, $\square, \diamond\}$.

3. $\nu \varepsilon^{\square} \alpha \vdash \nu \varepsilon \alpha \vdash \nu \varepsilon^{\diamond} \alpha$.

4. $\nu \mathcal{\varepsilon} \alpha, \nu \varepsilon^{\bullet} \beta \vdash \mathrm{I}^{\bullet} \alpha \beta$, for any $\bullet \in\{$ blank, $\square, \diamond\}$.

5. $\nu \varepsilon \alpha, \neg \nu \varepsilon^{\bullet} \beta \vdash \mathrm{O}^{*} \alpha \beta$, where $*=$ blank, $\diamond, \square$, for $\bullet=$ blank, $\square, \diamond$, respectively.

PROOF. 4. $\nu \varepsilon \alpha, \nu \varepsilon^{\bullet} \beta, \neg \mathrm{I}^{\bullet} \alpha \beta \stackrel{\mathrm{RN}}{\longrightarrow} \mathrm{E}^{*} \alpha \beta \stackrel{\mathrm{RI}_{1}}{\longrightarrow} \neg \nu \varepsilon^{\bullet} \beta \times$

5. $\nu \varepsilon \alpha, \neg \nu \varepsilon^{\bullet} \beta, \neg \mathrm{O}^{*} \alpha \beta \stackrel{\mathrm{RN}}{\longrightarrow} \mathrm{A}^{\bullet} \alpha \beta \stackrel{\mathrm{RI}_{1}}{\longrightarrow} \nu \varepsilon^{\bullet} \beta \times$ 
Similarly, we obtain a suitable counterpart of Lemma 5.1, where for any branch $B, l^{B}$ is the number of letters from $\mathbf{S L}$ occurring in singular formulas on $B$ :

Lemma 8.3. For any non-empty finite subset $\Phi$ of For $^{+}$and any "minimax" open tableau $T$ for $\Phi$, there is a model $\mathfrak{M}_{T} \in \operatorname{Mod}^{+}$such that $\mathfrak{M}_{T} \vDash \Phi$ and its cardinality is equal to $\min \left\{p_{\mathrm{e}}^{B}+l^{B}+1: B\right.$ is an open branch in $\left.T\right\}$.

Proof. Let $B$ be an open branch in $T$ such that $B$ has the smallest number $p_{\mathrm{e}}^{B}$ among all open branches in $T$. By definition of $T$, all formulas of $\Phi$ are on $B$. We construct the following model $\mathfrak{M}_{T}=\left\langle D, d^{s}, d, d^{\square}, d^{\diamond}\right\rangle$ from $\operatorname{Mod}^{+}$.

If $p_{\mathrm{e}}^{B}+l^{B}=0$, then $D:=\{0\}$ and for all $\bullet \in\{$ blank, $\square, \diamond\}, \alpha \in \mathbf{G L}$, and $\nu \in$ SL we put: $d^{\bullet}(\alpha):=\emptyset$ and $d^{S}(\nu):=0$.

If $p_{\mathrm{e}}^{B}+l^{B}>0$, then we put:

$$
\begin{aligned}
D:= & \{0\} \cup\left\{\imath \in \mathbf{A I}: \exists_{\alpha \in \mathbf{G L}}\ulcorner\imath \varepsilon \alpha\urcorner \text { is on } B\right\} \cup \\
& \left\{\nu \in \mathbf{S L}: \exists_{\alpha \in \mathbf{G L}}\ulcorner\nu \varepsilon \cdot \alpha\urcorner \text { is on } B, \text { for some } \bullet \in\{\text { blank, } \square, \diamond\}\right\} .
\end{aligned}
$$

Because $T$ is "mini-max", $\operatorname{Card}(D)=p_{\mathrm{e}}^{B}+l^{B}+1$ and for any $\imath \in \mathbf{S L} \cup \mathbf{A I}$ we have:

$$
\imath \in D \quad \text { iff } \quad \exists_{\alpha \in \mathbf{G L}}\left\ulcorner\imath \varepsilon^{\bullet} \alpha\right\urcorner \text { is on } B \text {, for some } \bullet \in\{\text { blank, } \square, \diamond\} .
$$

Moreover, for all $\alpha \in$ GL and $\bullet \in\{$ blank, $\square, \diamond\}$ we put:

$$
d^{\bullet}(\alpha):=\left\{\imath \in \mathbf{S L} \cup \mathbf{A I}:\left\ulcorner\imath \varepsilon^{\bullet} \alpha\right\urcorner \text { is on } B\right\} .
$$

So $0 \notin d^{\bullet}(\alpha)$. Moreover, for any $\nu \in \mathbf{S L}$ we put:

$$
d^{s}(\nu):= \begin{cases}\nu & \text { if } \nu \in D \\ 0 & \text { if } \nu \notin D\end{cases}
$$

$\mathfrak{M}_{T}:=\left\{D, d^{s}, d, d^{\square}, d^{\diamond}\right\}$ belongs to $\operatorname{Mod}^{+}$, because it satisfies condition $(\star)$, as shown in the proof of Lemma 5.1.

Now we show that for any $\varphi \in$ For $^{+}$we obtain: if $\varphi$ is on $B$, then $\mathfrak{M}_{T} \vDash \varphi$. The proof is by induction on the complexity of formulas.

Suppose that for some $\bullet \in\{$ blank, $\square, \diamond\},\left\ulcorner\nu \varepsilon^{\bullet} \alpha\right\urcorner$ is on $B$. Then $\nu \in D$, $d^{S}(\nu)=\nu$, and $\nu \in d^{\bullet}(\alpha)$. So $\mathfrak{M}_{T} \vDash \nu \varepsilon^{\bullet} \alpha$.

Suppose that $\left\ulcorner\neg \nu \varepsilon^{\bullet} \alpha\right\urcorner$ is on $B$, for some $\bullet \in\{$ blank, $\square, \diamond\}$. Then $\left\ulcorner\nu \varepsilon^{\bullet} \alpha\right\urcorner$ is not on $B$, since $B$ is open. So $\nu \notin d^{\bullet}(\alpha)$. Hence in both cases $d^{5}(\nu)=\nu$ and $d^{s}(\nu)=0$ we have $d^{s}(\nu) \notin d^{\bullet}(\alpha)$. So $\mathfrak{M}_{T} \not \models \nu \varepsilon^{\bullet} \alpha$ and $\mathfrak{M}_{T} \vDash \neg \nu \varepsilon^{\bullet} \alpha$.

The rest is as in the proof of Lemma 5.1. 
We also have soundness theorem (a counterpart of Theorem 4.1), completeness theorem (a counterpart of Theorem 5.2), and decidability (a counterpart of theorems 6.2 and 6.3). In the last case we replace merely a number $p_{\mathrm{e}}^{B}$ with a number $p_{\mathrm{e}}^{B}+l^{B}+1$; and also in the calculation of the number $\operatorname{Max}(\Psi, \varphi)$.

Its decidability is a result of the fact that relation $\models_{\mathrm{CQL}}$ is decidable. In addition, we would like to estimate cardinalities of models for For $^{+}$that are less than the cardinalities of models for CQL. It is known that for $\Psi \in$ $\mathcal{P}\left(\boldsymbol{F o r}_{\mathcal{L}^{+}}\right)$and $\varphi \in \operatorname{For}_{\mathcal{L}^{+}}$to check whether $\Psi \models_{\mathrm{CQL}} \varphi$ it is enough to examine all models whose cardinalities are not greater than $2^{k}$, where $k$ is a number of all monadic predicates from $\mathbf{P}_{\mathcal{L}}$ occurring in the set $\Psi \cup\{\varphi\}$. So the number of individual constants occurring in this set is not essential [see 12, Corollary 2]. ${ }^{11}$

\section{Aristotelian Modal Syllogisms}

\subsection{Semantics}

As we mentioned in the introduction, in $[4,14,15]$ the aim was to reconstruct Aristotelian modal syllogistic [1], where the pairs of formulas $\mathrm{I}^{\square} \alpha \beta$ and $\mathrm{I}^{\square} \beta \alpha$ (and so also $E^{\diamond} \alpha \beta$ and $E^{\diamond} \beta \alpha$ ) are equivalent. So in $[4,14,15]$ for models of the form $\left\langle D, d, d^{\square}, d^{\diamond}\right\rangle$, satisfying among others condition $(\star)$ from p. 4, a different interpretation of $\mathrm{I}^{\square}$ and $\mathrm{E}^{\diamond}$ we use:

$$
\begin{aligned}
& \mathfrak{M} \vDash \mathrm{I}^{\square} \alpha \beta \text { iff } d^{\square}(\alpha) \cap d^{\square}(\beta) \neq \emptyset, \\
& \mathfrak{M} \vDash \mathrm{E}^{\diamond} \alpha \beta \text { iff } \mathfrak{M} \not \models \mathrm{I}^{\square} \alpha \beta \text { iff } d^{\square}(\alpha) \cap d^{\square}(\beta)=\emptyset .
\end{aligned}
$$

Moreover, for some reasons given in $[4,14,15]$ another interpretation of $\mathrm{O}^{\square}$ and so of $A^{\diamond}$ were used:

$$
\begin{aligned}
& \mathfrak{M} \vDash \mathrm{O}^{\square} \alpha \beta \text { iff } d^{\square}(\alpha) \nsubseteq d^{\diamond}(\beta), \\
& \mathfrak{M} \vDash A^{\diamond} \alpha \beta \text { iff } \mathfrak{M} \not \models \mathrm{O}^{\square} \alpha \beta \text { iff } d^{\square}(\alpha) \subseteq d^{\diamond}(\beta) .
\end{aligned}
$$

\footnotetext{
${ }^{11}$ The latter becomes important if we consider a monadic predicate logic with equality. Then an examination of whether $\Psi=\mathrm{CQL} \varphi$ requires only the examination of all models with a cardinality not greater than $l+2^{k} \cdot \max \{n, 1\}$, where $k$ is a number of all monadic predicates occurring in the set $\Psi \cup\{\varphi\}, l$ (resp. $n$ ) is a number of all individual constants (resp. variables) occurring in the non-tautological equations from this set, and the tautological equations have the form $\ulcorner A=A\urcorner$. If in the set $\Psi \cup\{\varphi\}$ there are no variables then the above estimation can be reduced to $\max \{l, 1\}+\min \left\{2^{k}-1, q-\max \{l, 1\}\right\}$, where $q$ is the number of individual constants occurring in this set [see 12, p. 52].
} 
In $[4,14,15]$ various conditions on a denotation for models are imposed. For example, in $[14,15](\star)$ is assumed plus for all $\alpha, \beta \in \mathbf{G L}$ :

$$
\begin{aligned}
& d^{\square}(\alpha) \neq \emptyset, \\
& \text { if } d(\alpha) \cap d^{\diamond}(\beta) \neq \emptyset \text { then } d^{\diamond}(\alpha) \cap d(\beta) \neq \emptyset, \\
& \text { if } d(\alpha) \cap d(\beta) \neq \emptyset \text { then } d(\alpha) \cap d^{\square}(\beta) \neq \emptyset, \\
& \text { if } d(\alpha) \subseteq d^{\square}(\beta) \text { then } d^{\diamond}(\alpha) \subseteq d^{\diamond}(\beta) .
\end{aligned}
$$

Let $\operatorname{Mod}_{\mathrm{Ar}}$ be the class of all models satisfying conditions $(\star)$ and $\left(1^{\mathrm{Ar}}\right)-$ $\left(4^{\mathrm{Ar}}\right)$. For this class we construct in the standard way the relation $\models_{\mathrm{Ar}}$. Using the revised interpretation and the conditions $(\star),\left(1^{\mathrm{Ar}}\right)$, and $\left(2^{\mathrm{Ar}}\right)$ we obtain:

Claim 9.1. For all $\alpha, \beta \in \mathbf{G L}$ :

1. $\mathrm{A}^{\bullet} \alpha \beta \models \mathrm{Ar}_{\mathrm{Ar}} \neg \mathrm{O}^{*} \alpha \beta$ and $\mathrm{E}^{\bullet} \alpha \beta \models \mathrm{Ar}_{\mathrm{Ar}} \neg \mathrm{I}^{*} \alpha \beta$, where $*=$ blank, $\diamond, \square$, for $\bullet=$ blank, $\square, \diamond$, respectively

2. For any $\mathrm{X} \in\{\mathrm{A}, \mathrm{I}, \mathrm{E}, \mathrm{O}\}: \mathrm{X}^{\square} \alpha \beta=_{\mathrm{Ar}} \mathrm{X} \alpha \beta=_{\mathrm{Ar}} \mathrm{X} \diamond \alpha \beta$.

3. $\mathrm{I}^{\bullet} \alpha \beta \models \mathrm{Ar}_{\mathrm{Ar}} \mathrm{I}^{\bullet} \beta \alpha$ and $\mathrm{E} \bullet \alpha \beta \models_{\mathrm{Ar}} \mathrm{E} \bullet \beta \alpha$, for any $\bullet \in\{$ blank, $\square, \diamond\}$.

4. $={ }_{\mathrm{Ar}} \mathrm{I}^{\bullet} \alpha \alpha$, for any $\bullet \in\{$ blank, $\square, \diamond\} .{ }^{12}$

5. $\models$ Ar $\mathrm{A} \alpha \alpha$ and $\models{ }_{\mathrm{Ar}} \mathrm{A}^{\diamond} \alpha \alpha$.

6. $\mathrm{A}^{\bullet} \alpha \beta=_{\mathrm{Ar}} \mathrm{I}^{\bullet} \alpha \beta$ and $\mathrm{E}^{\bullet} \alpha \beta=_{\mathrm{Ar}} \mathrm{O}^{\bullet} \alpha \beta$, for any $\bullet \in\{$ blank, $\square, \diamond\}$.

7. $\forall A_{\mathrm{Ar}} A^{\square} \alpha \alpha$.

\subsection{Tableau Rules}

We use the rules (RIg) only for $\bullet \in\{$ blank, $\square$ \} and the rules (RC) only for - $\in\{$ blank, $\diamond\}$. For the revised interpretation of $I^{\square}, E^{\diamond}, \mathrm{O}^{\square}, \mathrm{A}^{\diamond}$ we describe tableau rules in Table 8.

However, the rules (RIg), for $\bullet \in\{$ blank, $\square\},\left(\mathrm{RI}_{1}^{\mathrm{Ar}}\right),(\mathrm{RIa}),(\mathrm{RN}),(\mathrm{RC})$, for $\bullet \in\{$ blank, $\square\}$, and $\left(\mathrm{RC}^{\mathrm{Ar}}\right)$ are not sufficient for a reconstruction of the theory from [14], since they do not reflect conditions $\left(1^{\mathrm{Ar}}\right)-\left(4^{\mathrm{Ar}}\right)$ imposed on models. These conditions are reflected by the rules in Table 9 . The rule $\left(1_{\mathrm{r}}^{\mathrm{Ar}}\right)$ says that $\left\ulcorner\mathcal{E}^{\square} \alpha\right\urcorner$ can be put in any place in a tableau, but must be a new index in the tableau.

\footnotetext{
${ }^{12}$ We find condition $\left(1^{\text {Ar }}\right)$ non-intuitive. Why should it be that 'Some thief must be a thief' is logically valid in both interpretations of $\mathrm{I}^{\square}$ (in the natural interpretation for $\models \mathrm{I}^{\square} a a$ we use both $(\star)$ and $\left.\left(1^{\mathrm{Ar}}\right)\right)$ ?
} 


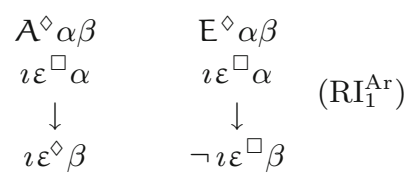

where $\imath$ is any index

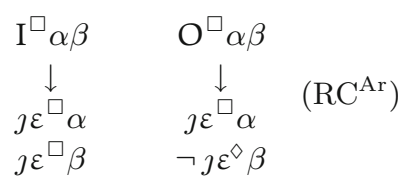

where $\jmath$ is a new index in a tableau

Table 8. Rules $\left(\mathrm{RI}_{1}^{\mathrm{a}}\right)$ and $\left(\mathrm{RC}^{\mathrm{a}}\right)$

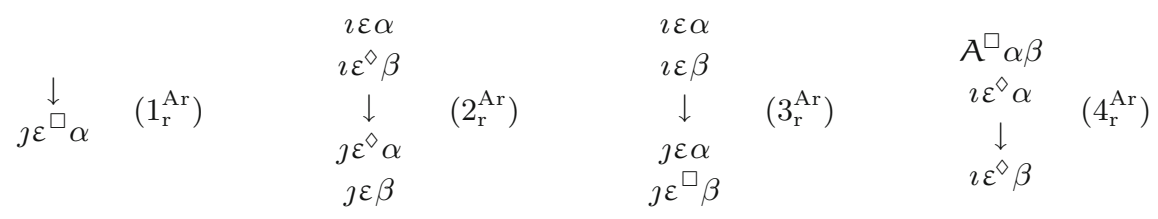

where $\imath$ is any index, but $\jmath$ is a new index in a tableau

Table 9. Additional rules

Using the above rules we obtain the following facts corresponding to those in Claim 9.1:

Claim 9.2. For all $\alpha, \beta \in \mathbf{G L}$ :

1. $A^{\bullet} \alpha \beta \mapsto_{\mathrm{Ar}} \neg \mathrm{O}^{*} \alpha \beta$ and $\mathrm{E}^{\bullet} \alpha \beta \mapsto_{\mathrm{Ar}} \neg \mathrm{I}^{*} \alpha \beta$, where $*=$ blank, $\diamond, \square$, for $\bullet=$ blank, $\square, \diamond$, respectively.

2. For any $\mathrm{X} \in\{\mathrm{A}, \mathrm{I}, \mathrm{E}, \mathrm{O}\}: \mathrm{X}^{\square} \alpha \beta \vdash_{\mathrm{Ar}} \mathrm{X} \alpha \beta \vdash_{\mathrm{Ar}} \mathrm{X}{ }^{\diamond} \alpha \beta$.

3. $\mathrm{I}^{\bullet} \alpha \beta \mapsto_{\mathrm{Ar}} \mathrm{I}^{\bullet} \beta \alpha$ and $\mathrm{E} \bullet \alpha \beta \vdash_{\mathrm{Ar}} \mathrm{E} \bullet \beta \alpha$, for any $\bullet \in\{$ blank, $\square, \diamond\}$.

4. $\vdash_{\mathrm{Ar}} \mathrm{I}^{\bullet} \alpha \alpha$, for any $\bullet \in\{$ blank, $\square, \diamond\}$.

5. $\vdash_{\mathrm{Ar}} \mathrm{A} \alpha \alpha$ and $\vdash_{\mathrm{Ar}} \mathrm{A}^{\diamond} \alpha \alpha$.

6. $A^{\bullet} \alpha \beta \vdash_{\mathrm{Ar}} \mathrm{I}^{\bullet} \alpha \beta$ and $\mathrm{E}^{\bullet} \alpha \beta \vdash_{\mathrm{Ar}} \mathrm{O}^{\bullet} \alpha \beta$, for any $\bullet \in\{$ blank, $\square, \diamond\}$.

PROOF. 3. $\mathrm{I}^{\square} \alpha \beta, \neg \mathrm{I}^{\square} \beta \alpha \stackrel{\mathrm{RC}^{\mathrm{Ar}}}{\longrightarrow} j \varepsilon^{\square} \alpha, j \varepsilon^{\square} \beta \stackrel{\mathrm{RN}}{\longrightarrow} \mathrm{E}^{\diamond} \beta \alpha \stackrel{\mathrm{RI}_{1}^{\mathrm{Ar}}}{\longrightarrow} \neg j \varepsilon^{\square} \beta \times$.

$\mathrm{I}^{\diamond} \alpha \beta, \neg \mathrm{I}^{\diamond} \beta \alpha \stackrel{\mathrm{RC}}{\longrightarrow} i \varepsilon \alpha, i \varepsilon^{\diamond} \beta \stackrel{2_{\mathrm{r}}^{\mathrm{Ar}}}{\longrightarrow} j \mathcal{E}^{\diamond} \alpha, j \varepsilon \beta \stackrel{\mathrm{RN}}{\longrightarrow} \mathrm{E}^{\square} \beta \alpha \stackrel{\mathrm{RI}_{1}^{\mathrm{Ar}}}{\longrightarrow} \neg j \mathcal{E}^{\diamond} \alpha \times$.

4. $\neg \mathrm{I}^{\square} \alpha \alpha \stackrel{\mathrm{RN}}{\longrightarrow} \mathrm{E}^{\diamond} \alpha \alpha \stackrel{1_{\mathrm{r}}^{\mathrm{Ar}}}{\longrightarrow} j \varepsilon^{\square} \alpha \stackrel{\mathrm{RI}_{1}^{\mathrm{Ar}}}{\longrightarrow} \neg j \varepsilon^{\square} \alpha \times$

$\neg \mathrm{I} \alpha \alpha \stackrel{\mathrm{RN}}{\longrightarrow} \mathrm{E} \alpha \alpha \stackrel{1_{\mathrm{r}}^{\mathrm{Ar}}}{\longrightarrow} j \varepsilon^{\square} \alpha \stackrel{\mathrm{RI}_{2}}{\longrightarrow} j \varepsilon \alpha \stackrel{\mathrm{RI}_{1}}{\longrightarrow} \neg j \varepsilon \alpha \times$

$\neg \mathrm{I}^{\diamond} \alpha \alpha \stackrel{\mathrm{RN}}{\longrightarrow} \mathrm{E}^{\square} \alpha \alpha \stackrel{1_{\mathrm{r}}^{\mathrm{Ar}}}{\longrightarrow} j \varepsilon^{\square} \alpha \stackrel{\mathrm{RI}_{2}}{\longrightarrow} j \varepsilon \alpha \stackrel{\mathrm{RI}_{2}}{\longrightarrow} j \mathcal{E}^{\diamond} \alpha \stackrel{\mathrm{RI}_{1}}{\longrightarrow} \neg j \mathcal{E}^{\diamond} \alpha \times$

6. $A^{\square} \alpha \beta, \neg \mathrm{I}^{\square} \alpha \beta \stackrel{\mathrm{RN}}{\longrightarrow} \mathrm{E}^{\diamond} \alpha \beta \stackrel{1_{\mathrm{r}}^{\mathrm{Ar}}}{\longrightarrow} j \mathcal{E}^{\square} \alpha \stackrel{\mathrm{RI}_{1}^{\mathrm{Ar}}}{\longrightarrow} \neg j \mathcal{E}^{\square} \beta \stackrel{\mathrm{RI}_{2}}{\longrightarrow} j \varepsilon \alpha \stackrel{\mathrm{RI}_{1}}{\longrightarrow} j \varepsilon^{\square} \beta \times$ $\mathrm{A}^{\diamond} \alpha \beta, \neg \mathrm{I}^{\diamond} \alpha \beta \stackrel{\mathrm{RN}}{\longrightarrow} \mathrm{E}^{\square} \alpha \beta \stackrel{1_{\mathrm{r}}^{\mathrm{Ar}}}{\longrightarrow} j \varepsilon^{\square} \alpha \stackrel{\mathrm{RI}_{1}^{\mathrm{Ar}}}{\longrightarrow} j \varepsilon^{\diamond} \beta \stackrel{\mathrm{RI}_{2}}{\longrightarrow} j \varepsilon \alpha \stackrel{\mathrm{RI}_{1}}{\longrightarrow} \neg j \varepsilon^{\diamond} \beta \times$ 
Under the unnatural interpretations of $\mathrm{I}^{\square}, \mathrm{E}^{\diamond}, \mathrm{O}^{\square}$ and $\mathrm{A}^{\diamond}$ which we have assumed here, which satisfy conditions $(\star),\left(1^{\mathrm{Ar}}\right)-\left(4^{\mathrm{Ar}}\right)$ we can reformulate and prove counterparts of the facts given in Sections 3-6.

\subsection{Soundness}

We prove that the revised relation $\vdash_{\mathrm{Ar}}$ is sound, i.e.:

Theorem 9.3 (Soundness). For all $\Psi \in \mathcal{P}($ For $)$ and $\varphi \in$ For:

$$
\text { if } \Psi \vdash_{\operatorname{Ar}} \varphi \text { then } \Psi \models \operatorname{Ar} \varphi \text {. }
$$

Proof. Assume that $\Psi \vdash_{\text {Ar }} \varphi$, i.e., there is a closed tableau $T$ for $\Psi \cup\{\neg \varphi\}$. Suppose towards contradiction that $\Psi \not \forall_{\mathrm{Ar}} \varphi$. Then there is a non-empty model $\mathfrak{M}=\left\langle D, d, d^{\square}, d^{\diamond}\right\rangle \in \operatorname{Mod}_{\text {Ar }}$ such that $\mathfrak{M} \vDash_{\text {Ar }} \Psi$, but $\mathfrak{M} \not \nvdash_{\text {Ar }} \varphi$; so $\mathfrak{M} \vDash_{\text {Ar }} \Psi \cup\{\neg \varphi\}$.

Note that all tableau rules of the types (NBE) and (RN) preserve truth and moreover at least one conclusion derived from the application of a rule of type (BE) preserves truth. Hence for some closed branch $B$ in $T$ all formulas from For which are in $B$ are true in $\mathfrak{M}$, because $\mathfrak{M} \vDash \Psi \cup\{\neg \varphi\}$. Let For $_{B}$ be the set of all formulas from For which are in $B$. So $\mathfrak{M} \vDash$ For $_{B}$.

If in $B$ no rule of type (RC) was used, then in $B$ there would be only formulas of $\mathbf{F o r}_{B}$. But this contradicts the fact that $B$ is closed. So we suppose that in $B$ at least once some rule of type $(\mathrm{RC})$ or $\left(\mathrm{RC}^{\mathrm{Ar}}\right)$ was used. Let $\Sigma_{B}^{1}$ be the set of auxiliary formulas in $B$ which we obtain by rules of $(\mathrm{RC})$ and $\left(\mathrm{RC}^{\mathrm{Ar}}\right)$. Let $I_{B}^{1}$ be the set of all indexes which appear in formulas from $\boldsymbol{\Sigma}_{B}^{1}$. We have $I_{B}^{1} \neq \emptyset$. Since we apply the rules of $(\mathrm{RC})$ and $\left(\mathrm{RC}^{\mathrm{Ar}}\right)$ only to formulas from For $_{B}$ and $\mathfrak{M} \vDash$ For $_{B}$, there is a valuation $v_{1}: I_{B}^{1} \rightarrow D$ such that $\mathfrak{M}, v_{1} \vDash \Sigma_{B}^{1}$. Then we suppose we could use also a rule of type $\left(1_{\mathrm{r}}^{\mathrm{Ar}}\right)-$ $\left(3_{\mathrm{r}}^{\mathrm{Ar}}\right)$. Let $\Sigma_{B}^{2}$ be the set of auxiliary formulas in $B$ which we obtain by rules of $\left(1_{\mathrm{r}}^{\mathrm{Ar}}\right)-\left(3_{\mathrm{r}}^{\mathrm{Ar}}\right)$. Let $I_{B}^{2}$ be the set of all indexes which appear in formulas from $\Sigma_{B}^{2}$. We have $I_{B}^{2} \neq \emptyset$. Since we apply the rules of $\left(1_{\mathrm{r}}^{\mathrm{Ar}}\right)-\left(3_{\mathrm{r}}^{\mathrm{Ar}}\right)$ only to formulas from $\boldsymbol{\Sigma}_{B}^{1}$ and $\mathfrak{M} \vDash \boldsymbol{\Sigma}_{B}^{1}$, by interpretation and the conditions $\left(1^{\mathrm{Ar}}\right)$ $\left(3^{\mathrm{Ar}}\right)$, there is a valuation $v_{2}: I_{B}^{2} \rightarrow D$ such that $\mathfrak{M}, v_{2} \vDash \Sigma_{B}^{2}$. Moreover, by interpretation and the condition $\left(4^{\mathrm{Ar}}\right)$, all other tableau rules preserve truth for $\mathfrak{M}$ and $v_{1} \cup v_{2}$. Hence, by induction on the length of $B$, we show that all formulas in $B$ are true in $\mathfrak{M}$, too. But this contradicts the fact that $B$ is closed.

\subsection{Compactness, Completeness, and Decidability}

By appropriate modification to the relevant proofs, we can obtain the counterparts of the facts from Sections 3-6. 
For all $\alpha, \beta \in \mathbf{G L}$ besides the formulas $\mathrm{T}_{\alpha}^{\square}$ and $\mathrm{T}_{\alpha}^{\diamond}$ we consider the following sentences from For $_{\mathcal{L}}$ :

$$
\begin{aligned}
1_{\alpha}^{\mathrm{Ar}} & :=\exists_{x} \alpha^{\square} x \\
2_{\alpha, \beta}^{\mathrm{Ar}} & :=\exists_{x}\left(\alpha^{\diamond} x \wedge \beta x\right) \supset \exists_{x}\left(\alpha x \wedge \beta^{\diamond} x\right) \\
3_{\alpha, \beta}^{\mathrm{Ar}} & :=\exists_{x}(\alpha x \wedge \beta x) \supset \exists_{x}\left(\alpha x \wedge \beta^{\square} x\right) \\
4_{\alpha, \beta}^{\mathrm{Ar}} & :=\forall_{x}\left(\alpha x \supset \beta^{\square} x\right) \supset \forall_{x}\left(\alpha^{\diamond} x \supset \beta^{\diamond} x\right)
\end{aligned}
$$

Moreover, for any $\Phi \in \mathcal{P}($ For $)$ we put:

$$
\begin{aligned}
\operatorname{Ar}_{\Phi}:= & \mathrm{T}_{\Phi} \cup\left\{1_{\alpha}^{\mathrm{Ar}} \in \text { For }_{\mathcal{L}}: \alpha \in \mathbf{G L}(\Phi)\right\} \\
& \cup\left\{k_{\alpha, \beta}^{\mathrm{Ar}} \in \text { For }_{\mathcal{L}}: k=2,3,4 \& \alpha, \beta \in \mathbf{G L}(\Phi)\right\} .
\end{aligned}
$$

We obtain the results corresponding to Lemma 1.4 and Theorem 1.5:

Lemma 9.4. For all $\Psi \in \mathcal{P}($ For $)$ and $\varphi \in$ For:

$$
\Psi=_{\mathrm{Ar}} \varphi \quad \text { iff } \quad \operatorname{Ar}_{\Psi \cup\{\varphi\}} \cup \mathbf{t}(\Psi) \models_{\mathrm{CQL}} \mathbf{t}(\varphi) .
$$

Theorem 9.5 (Compactness). For all $\Psi \in \mathcal{P}$ (For) and $\varphi \in$ For:

$$
\Psi \models_{\text {Ar }} \varphi \quad \text { iff } \text { there is } \Psi_{\text {fin }} \in \mathcal{P}_{\text {fin }}(\Psi) \text { such that } \Psi_{\text {fin }}==_{\text {Ar }} \varphi \text {. }
$$

We can easily specify the notion of a mini-max tableau for any non-empty finite subset $\Phi$ of For, where the rule $\left(1_{\mathrm{r}}^{\mathrm{Ar}}\right)$ we use only for some $\alpha$ that occurs in some formula from $\Phi$. For each such $\alpha$ we use this rule exactly once and the formula of the form $\left\ulcorner\jmath \varepsilon^{\square} \alpha\right\urcorner$ we put in the initial list. Moreover, we do not apply rules (RC) to formulas $\ulcorner\mathrm{I} \bullet \alpha \alpha\urcorner$, for any $\bullet \in\{$ blank, $\square, \diamond\}$.

For Completeness Theorem we use the following Completeness Lemma: ${ }^{13}$

Lemma 9.6. Let $\Phi$ be any non-empty finite subset of For and $T$ be any "mini-max" open tableau for $\Phi$. Then there is a model $\mathfrak{M}_{T} \in \operatorname{Mod}_{\mathrm{Ar}}$ such that $\mathfrak{M}_{T} \vDash \Phi$.

Proof. Let $B$ be an open branch in $T$. By definition, all formulas of $\Phi$ are in $B$. As in the proof of Lemma 5.1 we construct a model $\mathfrak{M}_{T}=\left\langle D, d, d^{\square}, d^{\diamond}\right\rangle$ from Mod $_{\mathrm{Ar}}$.

Now we show that $\mathfrak{M}_{T}$ is a model from $\operatorname{Mod}_{\mathrm{Ar}}$, i.e., for all $\alpha, \beta \in \mathbf{G L} \mathfrak{M}$ we have: $(\star)$ and $\left(1^{\mathrm{Ar}}\right)-\left(4^{\mathrm{Ar}}\right)$. Condition $(\star)$ we can show as in the proof of Lemma 5.1.

For $\left(1^{\mathrm{Ar}}\right)$ : By rule $\left(1_{\mathrm{r}}^{\mathrm{Ar}}\right)$, for any $\alpha$ that occurs in some formula from $\Phi$ on the initial list we have $\left\ulcorner\jmath \mathcal{E}^{\square} \alpha\right\urcorner$. Therefore $d^{\square}(\alpha) \neq \emptyset$.

\footnotetext{
${ }^{13}$ We will not be concerned here with the problem of minimal models, as we were in the case of Lemma 5.1.
} 
For $\left(2^{\mathrm{Ar}}\right)$ : Suppose that $d(\alpha) \cap d^{\diamond}(\beta) \neq \emptyset$. Then for some $\imath \in \mathbf{A I}$ formulas $\ulcorner\imath \varepsilon \alpha\urcorner$ and $\left\ulcorner\imath \varepsilon^{\diamond} \beta\right\urcorner$ are in $B$. In $B$ we also used the rule $\left(2_{\mathrm{r}}^{\mathrm{Ar}}\right)$. Hence for some new $\jmath \in \mathbf{A I}$ formulas $\left\ulcorner\jmath \mathcal{E}^{\diamond} \alpha\right\urcorner$ and $\ulcorner\jmath \varepsilon \beta\urcorner$ are in $B$. Therefore $d^{\diamond}(\alpha) \cap d(\beta) \neq \emptyset$.

For $\left(3^{\mathrm{Ar}}\right)$ : Suppose that $d(\alpha) \cap d(\beta) \neq \emptyset$. Then for some $\imath \in \mathbf{A I}$ formulas $\ulcorner\imath \varepsilon \alpha\urcorner$ and $\ulcorner\imath \varepsilon \beta\urcorner$ are in $B$. In $B$ we also used the rule $\left(3_{\mathrm{r}}^{\mathrm{Ar}}\right)$. Hence for some new $\jmath \in \mathbf{A I}$ formulas $\ulcorner\jmath \varepsilon \alpha\urcorner$ and $\left\ulcorner\jmath \varepsilon^{\square} \beta\right\urcorner$ are in $B$. So $d(\alpha) \cap d^{\square}(\beta) \neq \emptyset$.

For $\left(4^{\mathrm{Ar}}\right)$ : Suppose that we have $d(\alpha) \subseteq d^{\square}(\beta)$. Then, by $\left(1_{\mathrm{r}}^{\mathrm{Ar}}\right)$ and $\left(\mathrm{RI}_{2}\right)$, a formula $\left\ulcorner A^{\square} \alpha \beta\right\urcorner$ is in $B$. So we can use the rule $\left(4_{\mathrm{r}}^{\mathrm{Ar}}\right)$ to get $d^{\diamond}(\alpha) \subseteq d^{\diamond}(\beta)$, if $d^{\diamond}(\alpha) \neq \emptyset$, and in consequence some $\left\ulcorner\imath \varepsilon^{\diamond} \alpha\right\urcorner$ is in the branch.

Now we show that for any $\varphi \in$ For we obtain: if $\varphi$ is in $B$, then $\mathfrak{M}_{T} \vDash \varphi$. The proof is by induction on the complexity of formulas as in the proof of Lemma 5.1. We use the rules of $\left(\mathrm{RI}_{1}^{\mathrm{Ar}}\right)$ and of $\left(\mathrm{RC}^{\mathrm{Ar}}\right)$ only for $A^{\diamond}, \mathrm{E}^{\diamond}, \mathrm{I}^{\square}$, and $\mathrm{O}^{\square}$.

We have a counterpart of Theorem 5.2:

Theorem 9.7 (Completeness). For all $\Psi \subseteq$ For and $\varphi \in$ For:

$$
\text { if } \Psi=_{\mathrm{Ar}} \varphi \text {, then } \Psi \vdash_{\mathrm{Ar}} \varphi \text {. }
$$

Finally, we obtain a theorem corresponding to Theorem 6.1 :

Theorem 9.8 (Decidability). For all $\Psi \in \mathcal{P}_{\text {fin }}($ For $)$ and $\varphi \in$ For, the problem whether $\Psi \models \operatorname{Ar} \varphi$ is decidable.

Both theorems just give an alternative decision procedure for determining validity in the Thomason-Johnson semantics of Aristotelian modal syllogisms.

Acknowledgements. The research conducted by Tomasz Jarmużek which is presented in this paper was financed by National Science Centre, Poland, grant number: 2015/19/B/HS1/02478.

Open Access. This article is distributed under the terms of the Creative Commons Attribution 4.0 International License (http://creativecommons. org/licenses/by/4.0/), which permits unrestricted use, distribution, and reproduction in any medium, provided you give appropriate credit to the original author(s) and the source, provide a link to the Creative Commons license, and indicate if changes were made.

\section{References}

[1] Aristotle, Prior Analytics, Hackett, Indianapolis, 1989. Translated with introduction notes and commentary by R. Smith. 
[2] JARmużEK, T., Tableau system for logic of categorial propositions and decidability, Bulletin of the Section of Logic 37:223-231, 2008.

[3] Jarmużek, T., and A. Pietruszczak, Decidability methods for modal syllogisms, in A. Indrzejczak, J. Kaczmarek, and M. Zawidzki (eds.), Trends in Logic XIII, Eódź University Press, Łódź, 2014, pp. 95-112.

[4] Johnson, F., Models for modal syllogisms, Notre Dame Journal of Formal Logic 30(2):271-284, 1989. https://doi.org/10.1305/ndjfl/1093635084

[5] Kulicki, P., An axiomatisation of a pure calculus of names, Studia Logica 100(5):921946, 2012. https://doi.org/10.1007/s11225-012-9441-8

[6] Kulicki, P., On minimal models for pure calculi of names, Logic and Logical Philosphy 22(4):429-443, 2013. https://doi.org/10.12775/LLP.2013.023

[7] MCCall, S., Aristotle's Modal Syllogisms, Nort-Holland, Amsterdam, 1993.

[8] Pietruszczak, A., O logice tradycyjnej i rachunku nazw dopuszczajacym podstawienia nazw pustych (On traditional logic and calculi of names allowing substitutions of empty names), Ruch Filozoficzny 44:158-166, 1987.

[9] Pietruszczak, A., Standardowe rachunki nazw z funktorem Leśniewskiego (Pure calculi of names with Leśniewski's functor), Acta Universitatis Nicolai Copernici, Logika I:5-29, 1991.

[10] Pietruszczak, A., Bezkwantyfikatorowy rachunek nazw. Systemy i ich metateoria (Quantifier-free Calculus of Names. Systems and their Metatheory), Wydawnictwo Adam Marszałek, Toruń, 1991.

[11] Pietruszczak, A., Cardinalities of models for pure calculi of names, Reports on Mathematical Logic 28:87-102, 1994.

[12] Pietruszczak, A., Cardinalities of models for monadic predicate logic (with equality and individual constants), Reports on Mathematical Logic 30:49-64, 1996.

[13] Priest, G., An Introduction to Non-Classical Logic, 2th edition, Cambridge University Press, 2008. https://doi.org/10.1017/CBO9780511801174

[14] Thomason, S., Semantic analysis of the modal syllogistic, Journal of Philosophical Logic 22(2):111-128, 1993. https://doi.org/10.1007/BF01049258

[15] Thomason, S., Relational models for the modal syllogistic, Journal of Philosophical Logic 26:129-141, 1997. https://doi.org/10.1023/A:1004200616124

\author{
A. Pietruszczak, T. Jarmużek \\ Department of Logic \\ Nicolaus Copernicus University in Toruń \\ Toruń \\ Poland \\ pietrusz@umk.pl \\ T. JARMUŻEK \\ jarmuzek@umk.pl
}

